4-1-2010

\title{
Debye Series for Light Scattering by a Spheroid
}

Feng Xu

James A. Lock

Cleveland State University, j.lock@csuohio.edu

Cameron Tropea

Follow this and additional works at: https://engagedscholarship.csuohio.edu/sciphysics_facpub

Part of the Physics Commons

How does access to this work benefit you? Let us know!

\section{Publisher's Statement}

This paper was published in Journal of the Optical Society of America A: Optics Image Science and Vision and is made available as an electronic reprint with the permission of OSA. The paper can be found at the following URL on the OSA website: http://www.opticsinfobase.org/josaa/ abstract.cfm?URI=josaa-27-4-671. Systematic or multiple reproduction or distribution to multiple locations via electronic or other means is prohibited and is subject to penalties under law.

\section{Original Citation}

Xu, Feng, James A. Lock, and Cameron Tropea. "Debye Series for Light Scattering by a Spheroid." Journal of the Optical Society of America A: Optics Image Science and Vision 27 (2010): 671-686.

\section{Repository Citation}

Xu, Feng; Lock, James A.; and Tropea, Cameron, "Debye Series for Light Scattering by a Spheroid" (2010). Physics Faculty Publications. 9.

https://engagedscholarship.csuohio.edu/sciphysics_facpub/9

This Article is brought to you for free and open access by the Physics Department at EngagedScholarship@CSU. It has been accepted for inclusion in Physics Faculty Publications by an authorized administrator of EngagedScholarship@CSU. For more information, please contact library.es@csuohio.edu. 


\title{
Debye series for light scattering by a spheroid
}

\author{
Feng Xu, ${ }^{1, *}$ James A. Lock, ${ }^{2}$ and Cameron Tropea ${ }^{1}$ \\ ${ }^{1}$ Fachgebiet Strömungslehre und Aerodynamik, Technische Universität Darmstadt, \\ Petersenstraße 30, 64289 Darmstadt, Germany \\ ${ }^{2}$ Department of Physics, Cleveland State University, Cleveland, Ohio 44115, USA \\ *Corresponding author: f3_xu@yahoo.com
}

Received December 10, 2009; accepted January 9, 2010;

posted January 20, 2010 (Doc. ID 120568); published March 12, 2010

\begin{abstract}
The Debye series is developed for electromagnetic scattering by a spheroid in order to decompose the far-zone fields into various physical processes. The geometrical rainbow angle and supernumerary spacing parameter are determined from the Debye intensity by fitting the results to an Airy function and comparing them to their assumed values in ray optics and Airy theory, respectively. Eccentricity-related scattering phenomena including the rainbow's angular shift, the disappearance of the rainbow, and the rainbow-enhanced glory are quantitatively demonstrated and analyzed. (C) 2010 Optical Society of America

OCIS codes: $080.2710,290.4020$.
\end{abstract}

\section{INTRODUCTION}

The Lorenz-Mie theory (LMT) proposed a century ago by Mie [1] provides a rigorous way to describe the scattering of a linearly polarized plane wave by a homogeneous sphere. It forms the basis of a number of techniques for optical particle characterization in various fields [2-5]. However, most particles found in nature or produced in industrial processes are nonspherical. In addition, the plane-wave assumption is not appropriate for laser beams of width approaching or less than the particle size. For scientific design and optimization of elastic-scatteringbased optical instruments, it is therefore necessary to advance the knowledge about the interaction between a shaped laser beam and a nonspherical particle and to better understand the scattered fields. As one type of regularly shaped particle, the spheroid has the practical value of approximating many slightly deformed particles to first order, e.g., cells stretched by two counterpropagating laser beams in the initial and intermediary periods [6] and droplets subjected to aerodynamic drag and shear forces $[7,8]$, or under the action of inertial forces and falling at low Reynolds number [9], or freely oscillating in the fundamental mode in a gaseous medium [10].

Significant differences of scattering by a spheroid from that by a sphere have been experimentally observed and theoretically predicted. One important example is the farzone rainbow. When observed in the plane perpendicular to the beam polarization direction, the rainbow shifts back and forth as a function of the angle between the beam direction and the spheroid major axis. This effect was calculated by Möbius to first order in the eccentricity $e$ (with $e^{2}=1-(a / b)^{2}$ for a prolate spheroid and $e^{2}$ $=(a / b)^{2}-1$ for an oblate one, where $a$ and $b$ are the semimajor and semi-minor axis lengths, respectively) [11]. For large eccentricity, ray-tracing methods have to be used to determine the geometrical optics (GO) rainbow angle $[12,13]$. Alternatively, by filtering the Fourier transformed intensity calculated by the full Mie theory, the rainbow maximum as a function of $a / b$ can be obtained
[14]. However, the error in the filtered intensities for a certain order rainbow has to be evaluated. This can be achieved if the Debye series is extended to the spheroid. The second example showing the difference of scattering by a spheroid from that by a sphere is either the absence of the rainbow as the spheroid axis ratio increases into a particular interval, causing the lack of deflection of exiting rays for the Descartes rainbow, or the presence of a rainbow-enhanced glory as the rainbow appears in the backward or forward direction. In addition, when an oblate droplet is illuminated by a horizontally incident laser beam, the slight eccentricity causes a bending of the rainbow in the horizontal rainbow region. Increasing the eccentricity leads to a coalescence of the rays leaving the droplet in the same direction above and below its equatorial plane, creating the cusped diffraction catastrophe, a hyperbolic umbilic diffraction catastrophe, or a symbolic umbilic diffraction catastrophe for increasing $a / b$ [15-17]. All these eccentricity-related phenomena are demonstrated and analyzed in the present and companion papers [18] by use of Debye series developed in the context of electromagnetic scattering.

Before proceeding to these phenomena, a brief review of the theoretical development of spheroid scattering by use of the variable separation method is helpful. Asano and Yamamoto's extension of LMT from a sphere to a spheroid provides a rigorous way to describe plane-wave scattering by a homogeneous spheroid [19]. Their work was later developed into the generalized Lorenz-Mie theory (GLMT) for shaped laser beam incidence [20-22]. However, neither LMT nor GLMT sheds light on the physical mechanisms responsible for the scattering. In contrast, the Debye series allows a detailed view of the scattering by following the propagation of each partial wave inside the particle. The cooperation of a number of partial waves produces the scattering at various orders, and each order $p$ has a particular physical meaning. The scattering of order $p$ corresponds, in the sense of GO, to the transmitted rays experiencing $(p-1)$ internal reflec- 
tions. By use of the Debye series, the information level of each oscillation in the Mie scattering curve concerning one of the particle's properties can be judged so that an instrument can be designed in an optimal manner. Through a spherical wave expansion, the Debye series was developed to match LMT and GLMT for a homogeneous sphere [23-25]. Through a plane-wave expansion, the Debye series was developed to match LMT and GLMT for a homogeneous cylinder with a circular cross section $[26,27]$. As a further extension, the Debye series for a multilayered sphere and multilayered cylinder was derived in [28,29], respectively. The present paper contributes to the development of the Debye series for a homogeneous spheroid.

\section{THEORY}

Consider a monochromatic, arbitrarily oriented, and shaped beam incident on a spheroid of semi-major axis length $a$ and semi-minor axis length $b$. The beam has a well-defined description of the electromagnetic fields in its own Cartesian coordinates $O_{B}-X Y Z$ with the positive $Z$ axis indicating the propagation direction of the beam and the $X$ axis indicating the polarization direction of the electric field. The time-dependent part of the field is $e^{-i \omega t}$. Let the $x$ axis of a second particle-centered coordinate system lie in the plane formed by the axisymmetric axis of the spheroid $O_{P} z$ and the beam propagation direction $O_{P} Z^{\prime}$, which is a translation of the $O_{B} Z$ axis to the particle coordinates $O_{P}-x y z$ with the known coordinates of the beam center $O_{B}\left(x_{0}, y_{0}, z_{0}\right)$. Two beam angles are used to relate the two coordinate systems. One is the incident angle $\theta_{i}$ indicating the beam propagation direction in particle coordinates, and the other is the polarization angle $\varphi_{i}$ indicating the polarization direction of the electric field relative to the $O_{P}-x z$ plane. Figures $1(\mathrm{a})$ and $1(\mathrm{~b})$ give the examples of oblique incidence of a Gaussian beam on a prolate and oblate spheroid, respectively. With the aid of $\left(\theta_{i}, \varphi_{i}\right)$ and $\left(x_{0}, y_{0}, z_{0}\right)$, the spheroidal beam shape coefficients (or more briefly, spheroidal BSCs), $G_{n}^{m, T E}$ and $G_{n}^{m, T M}$, can be evaluated in the spheroidal coordinates $(\xi, \eta, \varphi)$ and multiplied by spheroidal wave functions as the incident field expansion [30].

The variable separation method gives the solution to the scalar wave equation in prolate spheroidal coordinates in the following form:

$$
\psi_{m n}=R_{|m| n}(c ; \xi) S_{|m| n}(c ; \eta) \exp (i m \varphi),
$$

where $c$ is defined by the multiplication of the wave number $k$ and the semifocal length of the spheroid $f=\left(a^{2}\right.$ $\left.-b^{2}\right)^{1 / 2}$, namely, $c=k f$, and $R_{m n}$ and $S_{m n}$ are the spheroidal radial and angular functions, respectively. The vector wave functions in spheroidal coordinates are generated through the vector operation:

$$
\begin{aligned}
& \mathbf{M}_{m n}=\nabla \times\left(\mathbf{r} \psi_{m n}\right), \\
& \mathbf{N}_{m n}=\frac{1}{k} \nabla \times \mathbf{M}_{m n} .
\end{aligned}
$$

We use the spheroidal radial functions of the third kind $R_{m n}^{(3)}$ and of the fourth kind $R_{m n}^{(4)}$ to denote an outgoing and

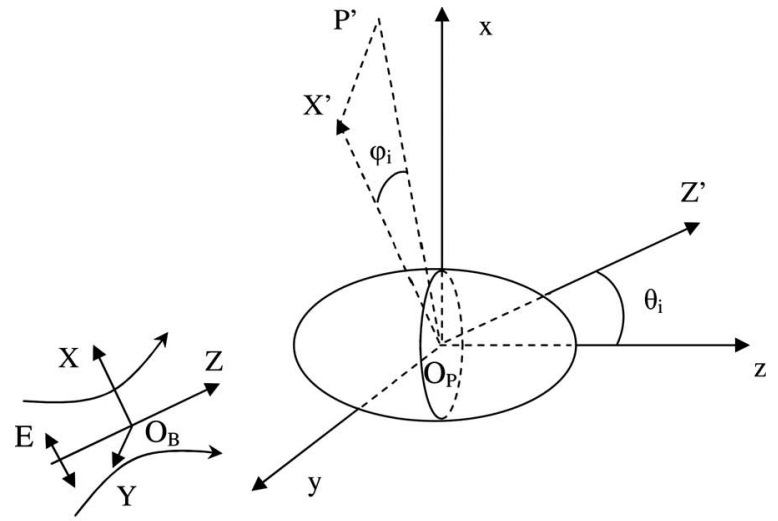

(a)

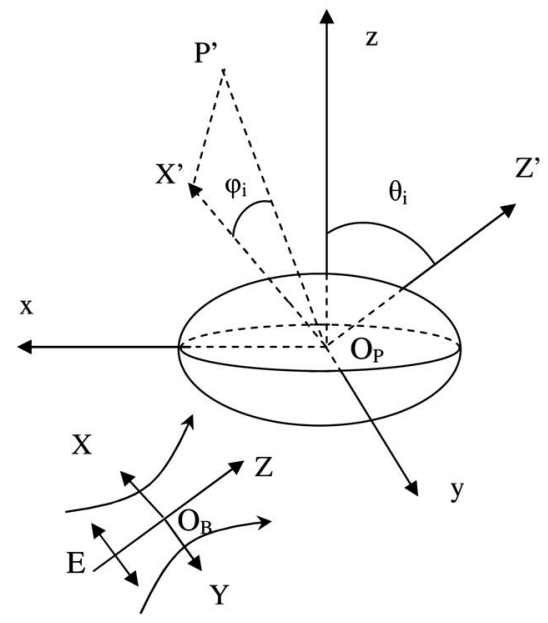

(b)

Fig. 1. Geometry for a spheroid illuminated by a Gaussian beam. The beam has its electric field polarized along the $X$ axis at its waist in its own coordinates $O_{B}-X Y Z$, with $\Theta$ and $\Phi$ being the angular and azimuthal components in the associated spherical coordinates while $\theta$ and $\varphi$ are the spherical coordinate system angles for the particle coordinates $O_{P}-x y z$. The $x$ axis lies in the plane formed by the $O_{P} z$ axis and the $O_{P} Z^{\prime}$ axis, which is a translation of the beam axis $O_{B} Z$ to the particle coordinates. The incidence angle $\theta_{i}$ is formed by the positive $O_{P} Z^{\prime}$ axis and the positive $O_{P} Z$ axis. Letting the $O_{P} X^{\prime}$ axis be the translation of the $O_{B} X$ axis in $O_{P}-x y z$, the polarization angle $\varphi_{i}$ measures the vibration direction of the electric field $\mathbf{E}$ with respect to the $O_{P}-x z$ plane (a clockwise rotation of $\varphi_{i}$ about the $O_{P} Z^{\prime}$ axis makes the $O_{P} X^{\prime}$ axis lie in the $O_{P}-x z$ plane). (a) Oblique incidence of the beam on a prolate spheroid. (b) Oblique incidence of the beam on an oblate spheroid.

incoming traveling wave, respectively. Accordingly, the vector spheroidal wave functions of the third type $\left(\mathbf{M}_{m n}^{(3)}, \mathbf{N}_{m n}^{(3)}\right)$ and fourth type $\left(\mathbf{M}_{m n}^{(4)}, N_{m n}^{(4)}\right)$ are generated, respectively.

\section{A. External Incidence at the Particle Surface}

Consider a nonmagnetic prolate spheroid of complex refractive index $M_{1}$ (region 1) embedded in a nonmagnetic and nonabsorbing medium of refractive index $M_{2}$ (region 2 ). The incident fields (denoted by the superscript " $i$ ") of a single spheroidal partial wave with the transverse electric (TE) polarization (the electric field vibrates perpendicular to the incidence plane $O_{P}-x z$ ) are given by 


$$
\begin{aligned}
\mathbf{E}_{m n_{0}, p=0}^{(i), T E} & =G_{n_{0}}^{m, T E} \mathbf{M}_{m n_{0}}^{(4)}\left(c_{2} ; \xi, \eta, \varphi\right), \\
\mathbf{H}_{m n_{0}, p=0}^{(i), T E} & =-\frac{i k_{2}}{\omega \mu_{2}}\left[G_{n_{0}}^{m, T E} \mathbf{N}_{m n_{0}}^{(4)}\left(c_{2} ; \xi, \eta, \varphi\right)\right],
\end{aligned}
$$

where $n_{0}$ is the partial wave number, $m$ is the azimuthal mode number, $G_{n_{0}}^{m, T E}$ is the TE partial wave amplitude, $c_{2}$ is the size parameter of the spheroid $\left(c_{2}=k_{2} f\right)$, and the or$\operatorname{der} p=0$ is assigned to the first interaction of the incident beam with the spheroid surface. Similarly, the incident fields of the single partial wave of the same order and mode but with the transverse magnetic (TM) polarization (the magnetic field vibrates perpendicular to the $O_{P}-x z$ plane) are described by

$$
\begin{aligned}
\mathbf{E}_{m n_{0}, p=0}^{(i), T M} & =G_{n_{0}}^{m, T M} \mathbf{N}_{m n_{0}}^{(4)}\left(c_{2} ; \xi, \eta, \varphi\right), \\
\mathbf{H}_{m n_{0}, p=0}^{(i), T M} & =-\frac{i k_{2}}{\omega \mu_{2}}\left[G_{n_{0}}^{m, T M} \mathbf{M}_{m n_{0}}^{(4)}\left(c_{2} ; \xi, \eta, \varphi\right)\right] .
\end{aligned}
$$

Designating $R_{n_{1}, n_{0}}^{m, 22}$ and $T_{n_{1}, n_{0}}^{m, 21}$ as the portions reflected back into region 2 and transmitted into the spheroid, respectively, when the partial wave of unit amplitude encounters the spheroid surface $\xi=\xi_{0}$, the entire reflected $(r)$ and transmitted $(t)$ electric fields of a single partial wave with the amplitude $G_{n_{0}}^{m, T E}$ can be described as the series sum over $n_{1}$ :

$$
\begin{aligned}
\mathbf{E}_{p=0}^{(r)}= & \sum_{n_{1}=|m|, n_{1} \neq 0}^{\infty}\left[\left(R_{n_{1}, n_{0}}^{m, 22, T E / T E} G_{n_{0}}^{m, T E}\right) \mathbf{M}_{m n_{1}}^{(3)}\left(c_{2} ; \xi, \eta, \varphi\right)\right. \\
& \left.+\left(R_{n_{1}, n_{0}}^{m, 22, T M / T E} G_{n_{0}}^{m, T E}\right) \mathbf{N}_{m n_{1}}^{(3)}\left(c_{2} ; \xi, \eta, \varphi\right)\right], \\
\mathbf{E}_{p=0}^{(t)}= & \sum_{n_{1}=|m|, n_{1} \neq 0}^{\infty}\left[\left(T_{n_{1}, n_{0}}^{m, 21, T E / T E} G_{n_{0}}^{m, T E}\right) \mathbf{M}_{m n_{1}}^{(4)}\left(c_{1} ; \xi, \eta, \varphi\right)\right. \\
& \left.+\left(T_{n_{1}, n_{0}}^{m, 21, T M / T E} G_{n_{0}}^{m, T E}\right) \mathbf{N}_{m n_{1}}^{(4)}\left(c_{1} ; \xi, \eta, \varphi\right)\right],
\end{aligned}
$$

respectively, where $c_{1}=k_{1} f$. The superscript $T E / T E$ indicates a TE wave generated from an incident TE wave, and the superscript $T M / T E$ indicates a TM field generated from an incident TE wave. Together with the TE wave generated from the TM incident wave, a crosspolarization effect is induced at the order $p=0$. Interchanging $\mathbf{M}_{m n}$ with $\mathbf{N}_{m n}$ in Eqs. (8) and (9) and multiplying by a prefactor $\left(-i k_{i} / \omega \mu_{i}\right)$ gives the magnetic field, with $i$ being 1 or 2 indicating the interior or exterior of the particle. Note that the incident fields in region 2 are radially incoming. One can get the explicit expressions for $R_{n_{1}, n_{0}}^{m, 22}$ and $T_{n_{1}, n_{0}}^{m, 2}$ for electromagnetic scattering by a sphere, coated sphere, and cylinder and scalar wave scattering by spheroid. But for electromagnetic wave scattering by a spheroid, the $R_{n_{1}, n_{0}}^{m, 22}$ and $T_{n_{1}, 21}^{m, 2}$ coefficients can only be numerically determined by solving large systems of coupled algebraic equations established from the boundary conditions describing the continuity of the tangential components of the electric and magnetic fields across the spheroid surface.
Summing the contribution over all incident TE and TM partial waves, the full reflected electric field for the order $p=0$ becomes

$$
\begin{aligned}
\mathbf{E}_{p=0}^{(r)}= & \sum_{m=-\infty}^{\infty} \sum_{n_{1}=|m|, n_{1} \neq 0}^{\infty}\left[W_{T E}^{(r)}(0) \mathbf{M}_{m n_{1}}^{(3)}\left(c_{2} ; \xi, \eta, \varphi\right)\right. \\
& \left.+W_{T M}^{(r)}(0) \mathbf{N}_{m n_{1}}^{(3)}\left(c_{2} ; \xi, \eta, \varphi\right)\right],
\end{aligned}
$$

where

$$
W_{T E}^{(r)}(0)=\sum_{n_{0}=|m|, n_{0} \neq 0}^{\infty}\left(R_{n_{1}, n_{0}}^{m, 22, T E / T E} G_{n_{0}}^{m, T E}+R_{n_{1}, n_{0}}^{m, 2, T E / T M} G_{n_{0}}^{m, T M}\right)
$$

$$
W_{T M}^{(r)}(0)=\sum_{n_{0}=|m|, n_{0} \neq 0}^{\infty}\left(R_{n_{1}, n_{0}}^{m, 22, T M / T E} G_{n_{0}}^{m, T E}+R_{n_{1}, n_{0}}^{m, 22, T M / T M} G_{n_{0}}^{m, T M}\right) .
$$

And the full transmitted electric field for the order $p=0$ becomes

$$
\begin{aligned}
\mathbf{E}_{p=0}^{(t)}= & \sum_{m=-\infty}^{\infty} \sum_{n_{1}=|m|, n_{1} \neq 0}^{\infty}\left[W_{T E}^{(t)}(0) \mathbf{M}_{m n_{1}}^{(4)}\left(c_{1} ; \xi, \eta, \varphi\right)\right. \\
& \left.+W_{T M}^{(t)}(0) \mathbf{N}_{m n_{1}}^{(4)}\left(c_{1} ; \xi, \eta, \varphi\right)\right],
\end{aligned}
$$

where

$$
W_{T E}^{(t)}(0)=\sum_{n_{0}=|m|, n_{0} \neq 0}^{\infty}\left(T_{n_{1}, n_{0}}^{m, 21, T E / T E} G_{n_{0}}^{m, T E}+T_{n_{1}, n_{0}}^{m, 21, T E / T M} G_{n_{0}}^{m, T M}\right)
$$

$$
W_{T M}^{(t)}(0)=\sum_{n_{0}=|m|, n_{0} \neq 0}^{\infty}\left(T_{n_{1}, n_{0}}^{m, 21, T M / T E} G_{n_{0}}^{m, T E}+T_{n_{1}, n_{0}}^{m, 21, T M / T M} G_{n_{0}}^{m, T M}\right) .
$$

\section{B. Internal Incidence at the Particle Surface}

When the wave is transmitted from the interior of the particle to the exterior, the vector wave functions of the third kind $\left(\mathbf{M}_{m n}^{(3)}, \mathbf{N}_{m n}^{(3)}\right)$ are used to denote the incident and transmitted fields, while vector wave functions of the fourth kind $\left(\mathbf{M}_{m n}^{(4)}, \mathbf{N}_{m n}^{(4)}\right)$ are used to denote the reflected fields. Accordingly, the full incident electric field for the interaction at the particle surface for the second time $(p=1)$ becomes 


$$
\begin{aligned}
\mathbf{E}_{p=1}^{(i)}= & \sum_{m=-\infty}^{\infty} \sum_{n_{1}=|m|, n_{1} \neq 0}^{\infty}\left\{\left[\sum_{n_{0}=|m|, n_{0} \neq 0}^{\infty}\left(T_{n_{1}, n_{0}}^{m, 21, T E / T E} G_{n_{0}}^{m, T E}+T_{n_{1}, n_{0}}^{m, 21, T E / T M} G_{n_{0}}^{m, T M}\right)\right] \mathbf{M}_{m n_{1}}^{(3)}\left(c_{1} ; \xi, \eta, \varphi\right)+\left[\sum _ { n _ { 0 } = | m | , n _ { 0 } \neq 0 } ^ { \infty } \left(T_{n_{1}, n_{0}}^{m, 21, T M / T E} G_{n_{0}}^{m, T E}\right.\right.\right. \\
& \left.\left.\left.+T_{n_{1}, n_{0}}^{m, 21, T M / T M} G_{n_{0}}^{m, T M}\right)\right] \mathbf{N}_{m n_{1}}^{(3)}\left(c_{1} ; \xi, \eta, \varphi\right)\right\}
\end{aligned}
$$

Taking into account the amplitudes of all incident TE and TM partial waves, the full reflected and transmitted electric fields of the order $p=1$ are

$$
\begin{aligned}
& \mathbf{E}_{p=1}^{(r)}=\sum_{m=-\infty}^{\infty} \sum_{n_{2}=|m|, n_{2} \neq 0}^{\infty}\left(\left\{\sum _ { n _ { 1 } = | m | , n _ { 1 } \neq 0 } ^ { \infty } \sum _ { n _ { 0 } = | m | , n _ { 0 } \neq 0 } ^ { \infty } \left[R_{n_{2}, n_{1}}^{m, 11, T E / T E}\left(T_{n_{1}, n_{0}}^{m, 21, T E / T E} G_{n_{0}}^{m, T E}+T_{n_{1}, n_{0}}^{m, 21, T E / T M} G_{n_{0}}^{m, T M}\right)+R_{n_{2}, n_{1}}^{m, 11, T E / T M}\left(T_{n_{1}, n_{0}}^{m, 21, T M / T E} G_{n_{0}}^{m, T E}\right.\right.\right.\right. \\
& \left.\left.\left.+T_{n_{1}, n_{0}}^{m, 21, T M / T M} G_{n_{0}}^{m, T M}\right)\right]\right\} \mathbf{M}_{m n_{2}}^{(4)}\left(c_{1} ; \xi, \eta, \varphi\right)+\left\{\sum _ { n _ { 1 } = | m | , n _ { 1 } \neq 0 } ^ { \infty } \sum _ { n _ { 0 } = | m | , n _ { 0 } \neq 0 } ^ { \infty } \left[R_{n_{2}, n_{1}}^{m, 11, T M / T E}\left(T_{n_{1}, n_{0}}^{m, 21, T E / T E} G_{n_{0}}^{m, T E}+T_{n_{1}, n_{0}}^{m, 21, T E / T M} G_{n_{0}}^{m, T M}\right)\right.\right. \\
& \left.\left.\left.+R_{n_{2}, n_{1}}^{m, 11, T M / T M}\left(T_{n_{1}, n_{0}}^{m, 21, T M / T E} G_{n_{0}}^{m, T E}+T_{n_{1}, n_{0}}^{m, 21, T M / T M} G_{n_{0}}^{m, T M}\right)\right]\right\} \mathbf{N}_{m n_{2}}^{(4)}\left(c_{1} ; \xi, \eta, \varphi\right)\right) \\
& \mathbf{E}_{p=1}^{(t)}=\sum_{m=-\infty}^{\infty} \sum_{n_{2}=|m|, n_{2} \neq 0}^{\infty}\left(\left\{\sum _ { n _ { 1 } = | m | , n _ { 1 } \neq 0 } ^ { \infty } \sum _ { n _ { 0 } = | m | , n _ { 0 } \neq 0 } ^ { \infty } \left[T_{n_{2}, n_{1}}^{m, 12, T E / T E}\left(T_{n_{1}, n_{0}}^{m, 21, T E / T E} G_{n_{0}}^{m, T E}+T_{n_{1}, n_{0}}^{m, 21, T E / T M} G_{n_{0}}^{m, T M}\right)+T_{n_{2}, n_{1}}^{m, 12, T E / T M}\left(T_{n_{1}, n_{0}}^{m, 21, T M / T E} G_{n_{0}}^{m, T E}\right.\right.\right.\right. \\
& \left.\left.\left.+T_{n_{1}, n_{0}}^{m, 21, T M / T M} G_{n_{0}}^{m, T M}\right)\right]\right\} \mathbf{M}_{m n_{2}}^{(3)}\left(c_{2} ; \xi, \eta, \varphi\right)+\left\{\sum _ { n _ { 1 } = | m | , n _ { 1 } \neq 0 } ^ { \infty } \sum _ { n _ { 0 } = | m | , n _ { 0 } \neq 0 } ^ { \infty } \left[T_{n_{2}, n_{1}}^{m, 12, T M / T E}\left(T_{n_{1}, n_{0}}^{m, 21, T E / T E} G_{n_{0}}^{m, T E}+T_{n_{1}, n_{0}}^{m, 21, T E / T M} G_{n_{0}}^{m, T M}\right)\right.\right. \\
& \left.\left.\left.+T_{n_{2}, n_{1}}^{m, 12, T M / T M}\left(T_{n_{1}, n_{0}}^{m, 21, T M / T E} G_{n_{0}}^{m, T E}+T_{n_{1}, n_{0}}^{m, 21, T M / T M} G_{n_{0}}^{m, T M}\right)\right]\right\} \mathbf{N}_{m n_{2}}^{(3)}\left(c_{2} ; \xi, \eta, \varphi\right)\right)
\end{aligned}
$$

respectively, where the reflected and transmitted portions of the single partial wave of unit amplitude are designated as $R_{n_{2}, n_{1}}^{m, 11}$ and $T_{n_{2}, n_{1}}^{m, 12}$ respectively.

Differing from order $p=0$, the incident fields at the $p$ $=1$ interface are radially outgoing in region 1 . But similar to order $p=0$, one cannot get the explicit expression for $R_{n_{2}, n_{1}}^{m, 11}$ and $T_{n_{2}, n_{1}}^{m, 12}$ for electromagnetic wave scattering by a spheroid. Therefore again these coefficients are numerically determined through boundary conditions, matching and solving sets of coupled algebraic equations.

Without loss of generality, the full incident electric field for an arbitrary order $p(p \geqslant 1)$ can be described in the following way:

$$
\begin{aligned}
\mathbf{E}_{p}^{(i)}= & \sum_{m=-\infty}^{\infty} \sum_{n_{p}=|m|, n_{p} \neq 0}^{\infty}\left[W_{T E}^{(i)}(p) \mathbf{M}_{m n_{p}}^{(3)}\left(c_{1} ; \xi, \eta, \varphi\right)\right. \\
& \left.+W_{T M}^{(i)}(p) \mathbf{N}_{m n_{p}}^{(3)}\left(c_{1} ; \xi, \eta, \varphi\right)\right],
\end{aligned}
$$

where $W_{T E}^{(i)}$ and $W_{T M}^{(i)}$ are given by

$$
\begin{aligned}
& W_{T E}^{(i)}(p)=\sum_{n_{p-1}=|m|, n_{p-1} \neq 0}^{\infty} \ldots \sum_{n_{0}=|m|, n_{0} \neq 0}^{\infty} V_{p}(1), \\
& W_{T M}^{(i)}(p)=\sum_{n_{p-1}=|m|, n_{p-1} \neq 0}^{\infty} \ldots \sum_{n_{0}=|m|, n_{0} \neq 0}^{\infty} V_{p}(2) .
\end{aligned}
$$

Here the two-element column vector $\mathbf{V}_{p}$ can be obtained via the following matrix operation:

$$
\begin{aligned}
\mathbf{V}_{p}= & {\left[\begin{array}{ll}
F_{n_{p}, n_{p-1}}^{m, T E / T E} & F_{n_{p}, n_{p-1}}^{m, T E / T M} \\
F_{n_{p}, n_{p-1}}^{m, T M / T E} & F_{n_{p}, n_{p-1}}^{m, T M / T M}
\end{array}\right] \cdots\left[\begin{array}{ll}
F_{n_{1}, n_{0}}^{m, T E / T E} & F_{n_{1}, n_{0}}^{m, T E / T M} \\
F_{n_{1}, n_{0}}^{m, T M / T E} & F_{n_{1}, n_{0}}^{m, T M / T M}
\end{array}\right] } \\
& \times\left[\begin{array}{c}
G_{n_{0}}^{m, T E} \\
G_{n_{0}}^{m, T M}
\end{array}\right]
\end{aligned}
$$

where $F=T^{21}$ for $n_{i}$ with $i=1$ and $F=R^{11}$ for $n_{i}$ with $i \geqslant 2$.

Similarly, the full reflected electric field can be described by

$$
\begin{aligned}
\mathbf{E}_{p}^{(r)}= & \sum_{m=-\infty}^{\infty} \sum_{n_{p+1}=|m|, n_{p+1} \neq 0}^{\infty}\left[W_{T E}^{(r)}(p) \mathbf{M}_{m n_{p+1}}^{(4)}\left(c_{1} ; \xi, \eta, \varphi\right)\right. \\
& \left.+W_{T M}^{(r)}(p) \mathbf{N}_{m n_{p+1}}^{(4)}\left(c_{1} ; \xi, \eta, \varphi\right)\right]
\end{aligned}
$$

where

$$
\begin{gathered}
W_{T E}^{(r)}(p)=W_{T E}^{(i)}(p+1), \\
W_{T M}^{(r)}(p)=W_{T M}^{(i)}(p+1) .
\end{gathered}
$$

The transmitted electric field can be described by

$$
\begin{aligned}
\mathbf{E}_{p}^{(t)}= & \sum_{m=-\infty}^{\infty} \sum_{n_{p+1}=|m|, n_{p+1} \neq 0}^{\infty}\left[W_{T E}^{(t)}(p) \mathbf{M}_{m n_{p+1}}^{(3)}\left(c_{2} ; \xi, \eta, \varphi\right)\right. \\
& \left.+W_{T M}^{(t)}(p) \mathbf{N}_{m n_{p+1}}^{(3)}\left(c_{2} ; \xi, \eta, \varphi\right)\right]
\end{aligned}
$$

where 


$$
\begin{aligned}
& W_{T E}^{(t)}(p)=W_{T E}^{(i)}(p+1), \\
& W_{T M}^{(t)}(p)=W_{T M}^{(i)}(p+1),
\end{aligned}
$$

but with the factor $F=T^{21}$ for $n_{i}$ with $i=1, F=R^{11}$ for $n_{i}$ with $2 \leqslant i \leqslant p$, and $F=T^{12}$ for $n_{i}$ with $i=p+1$ in the column vector $\mathbf{V}_{p+1}$. In each order of scattering the TM/TE crosspolarization part contains the term $F_{n_{p+1}, n_{p}}^{m, T M / T E}$ in $V_{p+1}(2)$, and the TE/TM cross-polarization part contains the term $F_{n_{R+1}, n_{p}}^{m, T E / T M}$ in $V_{p+1}(1)$.

Note that Subsections 2.A and 2.B focus on the contribution of a single partial wave to a certain order of scattering by tracing its successive interactions with the spheroid surface. A superposition of all partial waves results in the total field of that order. This approach provides a detailed view of the behavior of the single partial wave as it successively interacts with the spheroidmedium interface. In contrast, an overall view of a scattering order can be achieved by including all incident partial waves from the beginning order $p=0$ :

$$
\begin{aligned}
\mathbf{E}_{p=0}^{(i)}= & \sum_{m=-\infty}^{\infty} \sum_{n_{0}=|m|, n_{0} \neq 0}^{\infty}\left[G_{n_{0}}^{m, T E} \mathbf{M}_{m n_{0}}^{(4)}\left(c_{2} ; \xi, \eta, \varphi\right)\right. \\
& \left.+G_{n_{0}}^{m, T M} \mathbf{N}_{m n_{0}}^{(4)}\left(c_{2} ; \xi, \eta, \varphi\right)\right] .
\end{aligned}
$$

Then $\left(W_{T E}^{(r)}(0), W_{T M}^{(r)}(0)\right)$ and $\left(W_{T E}^{(t)}(0), W_{T M}^{(t)}(0)\right)$ are directly obtained by solving the boundary equations. Similarly, $\left(W_{T E}^{(r)}(p), W_{T M}^{(r)}(p)\right)$ and $\left(W_{T E}^{(t)}(p), W_{T M}^{(t)}(p)\right)$ for $p \geqslant 1$ are obtained successively by solving the boundary equations with the known coefficients from the order $(p-1)$ describing the incident fields for the order $p$.

\section{Debye Series for a Spheroid}

To deal with the full Mie problem for a spheroid, we expand the incident electric field and the internal electric field (denoted by the superscript "in"), using the spheroidal radial function of the first kind:

$$
\begin{aligned}
\mathbf{E}^{(i)}= & \sum_{m=-\infty}^{\infty} \sum_{n=|m|, n \neq 0}^{\infty}\left[G_{n}^{m, T E} \mathbf{M}_{m n}^{(1)}\left(c_{2} ; \xi, \eta, \varphi\right)\right. \\
& \left.+G_{n}^{m, T M} \mathbf{N}_{m n}^{(1)}\left(c_{2} ; \xi, \eta, \varphi\right)\right], \\
\mathbf{E}^{(\mathrm{in})}= & \sum_{m=-\infty}^{\infty} \sum_{n=|m|, n \neq 0}^{\infty}\left[D_{n}^{m} \mathbf{M}_{m n}^{(1)}\left(c_{1} ; \xi, \eta, \varphi\right)\right. \\
& \left.+C_{n}^{m} \mathbf{N}_{m n}^{(1)}\left(c_{1} ; \xi, \eta, \varphi\right)\right] .
\end{aligned}
$$

we expand the scattered electric field (denoted by the superscript $s$ ) using the spheroidal radial function of the third kind:

$$
\begin{aligned}
\mathbf{E}^{(s)}= & -\sum_{m=-\infty}^{\infty} \sum_{n=|m|, n \neq 0}^{\infty}\left[B_{n}^{m} \mathbf{M}_{m n}^{(3)}\left(c_{2} ; \xi, \eta, \varphi\right)\right. \\
& \left.+A_{n}^{m} \mathbf{N}_{m n}^{(3)}\left(c_{2} ; \xi, \eta, \varphi\right)\right] .
\end{aligned}
$$

Applying the boundary condition as described in Subsection 2.A for the Debye series evaluation, a large system of coupled algebraic equations is obtained and can be solved numerically for the partial wave scattering amplitudes $\left(A_{n}^{m}, B_{n}^{m}\right)$ and the interior amplitudes $\left(C_{n}^{m}, D_{n}^{m}\right)[22]$.
Referring to the proof in Appendix A, the Debye series and GLMT coefficients for the scattered fields can be related by

$$
\begin{aligned}
& A_{n}^{m}=\frac{1}{2}\left[G_{n}^{m, T M}-W_{T M}^{(r)}(0)-\sum_{p=1}^{\infty} W_{T M}^{(t)}(p)\right], \\
& B_{n}^{m}=\frac{1}{2}\left[G_{n}^{m, T E}-W_{T E}^{(r)}(0)-\sum_{p=1}^{\infty} W_{T E}^{(t)}(p)\right],
\end{aligned}
$$

where the first term describes the diffraction of the incident wave around the spheroid after being summed over $m$ and $n$, the second term represents the fields externally reflected from the spheroid surface, and the individual components of the series correspond to the contribution by the partial waves experiencing $(p-1)$ internal reflections.

The Debye series of the interior amplitudes is related to $\left(C_{n}^{m}, D_{n}^{m}\right)$ by

$$
\begin{aligned}
& C_{n}^{m}=W_{T M}^{(t)}(0)+\sum_{p=1}^{\infty} W_{T M}^{(r)}(p), \\
& D_{n}^{m}=W_{T E}^{(t)}(0)+\sum_{p=1}^{\infty} W_{T E}^{(r)}(p) .
\end{aligned}
$$

\section{Degeneration to the Case of a Sphere}

As the spheroid axis ratio $a / b$ tends to unity, the spheroidal coordinate system reduces to the spherical coordinate system, and Eq. (1) becomes

$$
\psi_{m n}=z_{n}(r) P_{|m| n}(\eta) \exp (i m \phi),
$$

where $z_{n}$ is a spherical Bessel function and $P_{m n}$ is an associated Legendre function.

As the spheroid becomes a sphere, all the $\mathrm{R}$ and $\mathrm{T}$ matrices (with elements $R_{n_{p}, n_{p-1}}^{m}$ and $T_{n_{p}, n_{p-1}}^{m}$ ) that were nondiagonal in the partial wave number for a spheroid now become diagonal for a sphere. As a function of $z_{n}$, the Debye series becomes independent of the azimuthal mode and equal to its counterpart in Mie-Debye scattering [31]. The spheroidal BSCs degenerate to the spherical BSCs with the time dependence $e^{-i \omega t}$, namely, $G_{n}^{m, T M}$ $\rightarrow c_{c w} g_{n}^{m, T M}$ and $G_{n}^{m, T E} \rightarrow c_{c w} i g_{n}^{m, T E}$. Moreover, no cross polarization is induced, since $F_{n_{p}, n_{p-1}}^{m, T E / T M}=F_{n_{p}, n_{p-1}}^{m, T M / T E}=0$. Thus Eqs. (20) and (21) for the incident fields become

$$
\begin{gathered}
W_{T E}^{(i)}(p)=c_{c w} i g_{n}^{m, T E} T_{n}^{21, T E}\left(R_{n}^{11, T E}\right)^{p-1}, \\
W_{T M}^{(i)}(p)=c_{c w} g_{n}^{m, T M} T_{n}^{21, T M}\left(R_{n}^{11, T M}\right)^{p-1},
\end{gathered}
$$

Eqs. (24) and (25) for the reflected fields become

$$
\begin{aligned}
& W_{T E}^{(r)}(p)=c_{c w} i g_{n}^{m, T E} T_{n}^{21, T E}\left(R_{n}^{11, T E}\right)^{p}, \\
& W_{T M}^{(r)}(p)=c_{c w} g_{n}^{m, T M} T_{n}^{21, T M}\left(R_{n}^{11, T M}\right)^{p},
\end{aligned}
$$

and Eqs. (27) and (28) for transmitted fields become

$$
W_{T E}^{(t)}(p)=c_{c w} i g_{n}^{m, T E} T_{n}^{21, T E}\left(R_{n}^{11, T E}\right)^{p-1} T_{n}^{12, T E},
$$




$$
W_{T M}^{(t)}(p)=c_{c w} g_{n}^{m, T M} T_{n}^{21, T M}\left(R_{n}^{11, T M}\right)^{p-1} T_{n}^{12, T M},
$$

where the partial wave amplitude of an incident planewave $c_{c w}$ is

$$
c_{c w}=i^{n+1} \frac{2 n+1}{n(n+1)} .
$$

Accordingly, Eqs. (33) and (34) become

$$
\begin{aligned}
A_{n}^{m}= & \frac{1}{2} c_{c w} g_{n}^{m, T M}\left[1-R_{n}^{22, T M}-\sum_{p=1}^{\infty}\left(T_{n}^{21, T M}\right)\right. \\
& \left.\times\left(R_{n}^{11, T M}\right)^{p-1} T_{n}^{12, T M}\right], \\
B_{n}^{m}= & \frac{1}{2} i c_{c w} g_{n}^{m, T E}\left[1-R_{n}^{22, T E}-\sum_{p=1}^{\infty}\left(T_{n}^{21, T E}\right)\right. \\
& \left.\times\left(R_{n}^{11, T E}\right)^{p-1} T_{n}^{12, T E}\right] .
\end{aligned}
$$

Similarly, Eqs. (35) and (36) become

$$
\begin{aligned}
& C_{n}^{m}=c_{c w} g_{n}^{m, T M} \sum_{p=1}^{\infty}\left(T_{n}^{21, T M}\right)\left(R_{n}^{11, T M}\right)^{p-1}, \\
& D_{n}^{m}=i c_{c w} g_{n}^{m, T E} \sum_{p=1}^{\infty}\left(T_{n}^{21, T E}\right)\left(R_{n}^{11, T E}\right)^{p-1} .
\end{aligned}
$$

Since $A_{n}^{m}=c_{c w} g_{n}^{m, T M} a_{n}, B_{n}^{m}=c_{c w} i g_{n}^{m, T E} b_{n}, C_{n}^{m}=c_{c w} g_{n}^{m, T M} c_{n}$, and $D_{n}^{m}=c_{c w} i g_{n}^{m, T E} d_{n}\left(a_{n}-d_{n}\right.$ are the field coefficients in the Mie theory), the special case of the Debye series for a sphere [32] is recovered when the common factors are removed from Eqs. (45)-(48).

Though in Section 2 we present the Debye series formalism only for a prolate spheroid, all the equations and expressions pertaining to prolate spheroidal coordinates can be converted to their counterparts in an oblate system by replacing the size parameter $c_{2}$ by $-i c_{2}\left(c_{1}\right.$ by $\left.-i c_{1}\right)$ and the spheroidal radial coordinate $\xi$ by $i \xi$.

\section{NUMERICAL RESULTS}

First, the sum of spheroid Debye series is compared with the total spheroid amplitude for a number of spheroid sizes with $c_{2}$ less than $\sim 80$ using a MATLAB code with double-precision arithmetic (64 bits). Namely, Eqs. (33) and (34) were validated numerically. The precision is found up to four digits after the decimal point. Moreover, it was verified that as $a / b \rightarrow 1$ the Debye series for a sphere is reproduced. Following these checks, the Debye series was employed to explore light scattering by a spheroidal water droplet for various orders, with emphasis on the rainbow observed with $\mathrm{TE}$ wave scattering. The incident wave is assumed to be planar and has its electric field vibrating perpendicular to the incidence plane $O_{P}-x z$. The refractive index of the droplet relative to the medium is $M=M_{1} / M_{2}=1.334+1.2 \times 10^{-9} \mathrm{i}$ at the wavelength $\lambda=0.5145 \mu \mathrm{m}$ [33]. Note that in the context of GLMT and Debye series for the spheroid, the intensity is described in the spheroid coordinates $(\xi, \eta, \varphi)$ where $\eta$ $=\cos (\theta)$ for the far-zone case. However, for convenience of rainbow analysis, the scattering is measured with respect to the beam propagation direction in the spherical coordinates $(R, \Theta, \Phi)$ associated with the beam coordinates $O_{B}-X Y Z$. Thus, a conversion relation $I\left(\Theta, \Phi=0^{\circ}\right)=I(\theta$ $\left.-\theta_{i}, \varphi=0^{\circ}\right)$ is used for the intensity plot in the $O_{P}-x z$ plane. In addition, for all numerical results in the present paper the scattering is calculated on an angular grid of $\Delta \theta=0.2^{\circ}$.

\section{A. Rainbow for End-On Wave Incidence}

In the full Mie intensity, the visibility of the rainbow is reduced as the particle size decreases to a few micrometers. However, in the context of the Debye series, a clear view of separate order rainbows is still available in this case. Before demonstrating the spheroid scattering, sphere scattering is first discussed for the purposes of (i) testing the procedures developed for spheroid rainbow analysis and (ii) checking two basic assumptions made for the sphere rainbow analysis by Airy theory.

\section{Sphere}

We decompose the Mie scattering for a spherical water droplet of radius $a=5.0 \mu \mathrm{m}$ into the first five orders of scattering ( $p$ from 0 to 4 ) and illustrate the results in Fig. 2(a). To overcome the divergence when the infinite series over partial waves is calculated for the individual processes of diffraction and external reflection, their contributions are summed for the order $p=0$. For comparison, Fig. 2(b) gives the GO decomposition of scattering by the same sphere, where the Fraunhofer diffracted field is superimposed on the $p=0$ order GO intensity in the forward angular range $0^{\circ} \leqslant \Theta \leqslant 20^{\circ}$. In GO, the stationary deflection of the emergent rays with respect to the incidence angle marks the position of the Descartes rainbow $\Theta_{D}$, where the two-ray-zero-ray transition causes the discontinuity of the intensity curve and the intensity infinity due to the infinitely dense emergent rays. In wave theory the intensity in the vicinity of the primary rainbow peak is assumed to be proportional to the square of the Airy function $\operatorname{Ai}(-u)$ [34]:

$$
I(\Theta) \propto \mathrm{Ai}^{2}\left\{-\alpha^{2 / 3}\left(\Theta-\Theta_{\mathrm{D}}^{p}\right) /\left(h_{\mathrm{D}}^{p}\right)^{1 / 3}\right\},
$$

where $\alpha$ is the sphere's size parameter $\left(\alpha=k_{1} \alpha\right)$. The two parameters $\Theta_{\mathrm{D}}$ and $h_{\mathrm{D}}$ in Eq. (49) are widely assumed in the Airy rainbow description to have the following values. The scattering angle corresponding to $\mathrm{Ai}(0)$ is the Descartes rainbow $\Theta_{\mathrm{D}}$ which can be analytically determined for a sphere by GO [35]:

$$
\Theta_{\mathrm{D}}^{p}=(p-1) \pi+2 \Theta_{\mathrm{D}}^{i}-2 p \Theta_{\mathrm{D}}^{t},
$$

where

$$
\cos \left(\Theta_{\mathrm{D}}^{i}\right)=\left[\left(M_{r}^{2}-1\right) /\left(p^{2}-1\right)\right]^{1 / 2},
$$




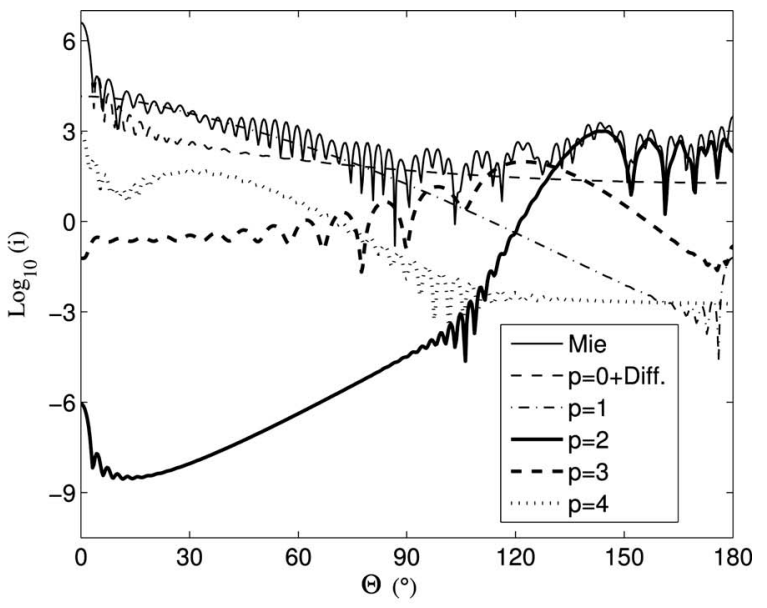

(a)

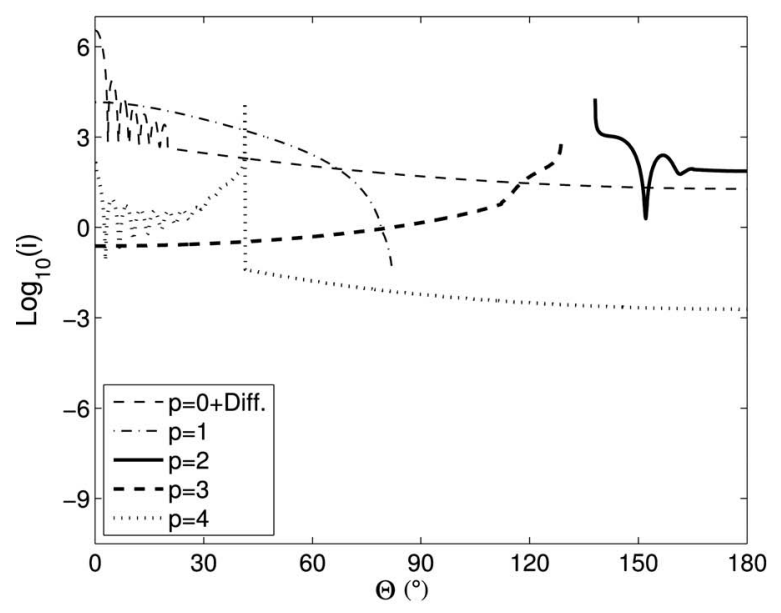

(b)

Fig. 2. (a) Mie scattering in the $\varphi=0^{\circ}$ plane and its Debye series decomposition into the first five orders $(p=0-4)$. The incident plane wave has the wavelength $\lambda=0.5145 \mu \mathrm{m}$, and its electric field is polarized in the $y$ direction. The spherical water droplet has a radius $r_{s}=5.0 \mu \mathrm{m}$ and relative refractive index $M=1.334$ $+1.2 \mathrm{e}-9 \mathrm{i}$. (b) GO decomposition of scattering by the same sphere.

$$
\sin \left(\Theta_{\mathrm{D}}^{t}\right)=\frac{\sin \left(\Theta_{\mathrm{D}}^{i}\right)}{M_{r}}
$$

where the subscript $r$ of $M$ indicates the real part of the relative refractive index of the particle and $\Theta_{\mathrm{D}}^{i}$ and $\Theta_{\mathrm{D}}^{t}$ are the incidence and refraction angles for the Descartes ray, respectively. The supernumerary spacing parameter $h_{\mathrm{D}}$ describes the cubic wave front exiting the spheroid in the vicinity of the Descartes ray and is evaluated in Airy theory [36] by

$$
h_{\mathrm{D}}=\frac{\left[\left(p^{2}-1\right)^{2}\left(p^{2}-M_{r}^{2}\right)^{1 / 2}\right]}{\left[p^{2}\left(M_{r}^{2}-1\right)^{3 / 2}\right]} .
$$

To check the accuracy of these assumptions, we assume that the Airy function description of the rainbow in the form of Eq. (49) is still valid. However, a variable geometrical rainbow angle $\Theta_{b}$ and a variable supernumerary spacing parameter $h_{\mathrm{b}}$ are introduced and determined in the following way: since the GO rainbow appears at $u=0$ with $\operatorname{Ai}(-u)=0.35503$ while the first maximum occurs at $u=1.01879$ with $\operatorname{Ai}(-u)=0.53566$, the ratio of the intensity at the GO rainbow angle to the intensity maximum is 0.4393. Applying the intensity ratio law, $\Theta_{\mathrm{b}}$ can be determined from the rainbow maximum in the Debye graph. Thus, for the spherical droplet of radius $5.0 \mu \mathrm{m}$ (or size parameter $\alpha=61.06) \Theta_{\mathrm{b}}$ is determined as $137.628^{\circ}$ from the Debye intensity curve for $p=2$, which is a deviation of $0.439^{\circ}$ from $\Theta_{\mathrm{D}}=138.067^{\circ}$. Similarly, $\Theta_{\mathrm{b}}$ for the rainbow of the second order $(p=3)$ is determined as $113.230^{\circ}$, which is a deviation of $15.617^{\circ}$ from $\Theta_{\mathrm{D}}=128.847^{\circ}$. Alternatively, both $\Theta_{\mathrm{b}}$ and $h_{\mathrm{b}}$ can be obtained through fitting the Debye scattering pattern to an Airy function as in Eq. (49). By use of the Simplex algorithm [37], the best fit to the main rainbow peak of the $p=2$ Debye intensity curve is achieved by using $\Theta_{\mathrm{b}}=137.533^{\circ}\left(h_{\mathrm{b}}=4.691\right)$, which is a deviation of $0.533^{\circ}$ from $\Theta_{\mathrm{D}}$. From the Debye intensity curve of $p=3, \Theta_{\mathrm{b}}$ is determined as $134.644^{\circ}\left(h_{\mathrm{b}}=35.481\right)$, which is a deviation of $5.796^{\circ}$. These calculations show that for a small particle size the true intensity distribution around the rainbow is distorted from the classical Airy curve parameterized by $\Theta_{\mathrm{D}}$ and $h_{\mathrm{D}}$. These distortions take the form of a shifting of the entire Airy function away from the Descartes angle, and the shape is stretched or compressed so that $h_{\mathrm{b}}$ 's value is different from $h_{\mathrm{D}}$ 's.

There are three possible reasons causing the GO rainbow angle and supernumerary spacing parameter to deviate from their assumed values for the particle size of a few micrometers. First, Airy theory in the form of Eq. (49) assumes a constant amplitude of the electric field in the virtual cubic wave front. However, to perform the Airy integral in a strict sense, the phase in the sphere exit plane has to be multiplied by the amplitude that has the Fresnel coefficients expressed in the Debye series form $T_{21}\left(R_{11}\right)^{p-1} T_{12}$. The Fresnel coefficients differ slightly at the angles of incidence of the two supernumerary rays. This effect yields the electric field $E=\mathrm{Ai}+i l_{p} \mathrm{Ai}^{\prime} / x^{1 / 3}$ in the vicinity of rainbow angles where $l_{p=2}=0.473$ and $l_{p=3}$ $=1.189$ for $M=1.334$ and $a=5.0 \mu \mathrm{m}$ [38]. The mixture of $\mathrm{Ai}$ and $\mathrm{Ai}^{\prime}$ causes error in the GO rainbow estimation from Eq. (49) when the size parameter $\alpha$ is small. As $\alpha$ becomes extremely large (say, $\alpha>5000$ [35]), the contribution of $\mathrm{Ai}^{\prime}$ becomes smaller with respect to $\mathrm{Ai}$ and $\left(\Theta_{\mathrm{D}}, h_{\mathrm{D}}\right)$ used for Eq. (49) become more accurate. Second, in the physical sense the Airy integral is an integral over a continuous range of impact parameters from negative infinity to infinity, whereas the Mie amplitude is a summation over a finite number of discrete partial waves. Sufficient approximation accuracy also requires the particle size to be large so that the partial wave sum approaches integration over a continuous range of impact parameters. The third possible reason for the deviation involves the surface waves improperly handled in Airy theory.

To determine the main reason for the deviation, the $\mathrm{Ai}^{\prime}$ correction is included into the Airy function fitting the Debye intensity. The reconstructed intensity graph from the numerically determined parameter set $\left(\Theta_{\mathrm{b}}, h_{\mathrm{b}}\right)$ indicates that the addition of the $\mathrm{Ai}^{\prime}$ correction raises the intensity minima closer to the Debye graph. However, it helps little in reducing the deviation $\left|\Theta_{b}-\Theta_{D}\right|$, since the intensities around the minimum play a limited role when the Sim- 
plex algorithm evaluates the fitting error. Therefore the constant amplitude assumption of the electric field causes little influence on the $\Theta_{\mathrm{b}}$ determination for TE wave scattering by the two methods. We then ascribe the deviation of the Airy approximation to the Mie intensity by the integral of impact parameters from negative infinity to infinity. This approximation loses accuracy for small particle size, since there are fewer partial waves to add up in the Mie theory and they are insufficient to be well approximated by the infinite impact range assumption in Airy theory. Moreover, the propagation of surface waves created by the edge-impacting rays with nearly grazingincidence angle is not strictly accounted for in Airy theory. Its contribution for an extremely large particle size is negligible compared with the contribution of real rays. However, for small particle sizes of a few micrometers, the influence of edge-impacting rays is no longer insignificant. They coherently superimpose on the contribution of the real rays and distort the intensity distribution around the rainbow. In Fig. 2(a) the surface wave effect can be observed in four places of the $p=2$ order intensity curve. Surface waves are formed after the $90^{\circ}$ grazing impact parameter ray at both the top and bottom of the sphere. These surface waves are counterpropagating. This gives rise to (i) constructive interference of the two counterpropagating surface waves near $0^{\circ}$. (ii) Between $10^{\circ}$ and $90^{\circ}$ the intensity is surface wave dominated. (iii) At about $105^{\circ}$ is the interference between the complex ray of the rainbow and one of the surface waves. (iv) The oscillations of the Airy function are continued past $165.768^{\circ}$ by the surface wave from the bottom of the sphere. But these oscillations are distorted by interference with surface waves from the top of the sphere (making this essentially a three-ray region). This interference can be seen in the doubling of the second to last relative maximum before $180^{\circ}$ and the shoulder on the third to last relative maximum. This interference with the surface waves from the top of the sphere is probably a big contribution to the difference between the Debye intensity and the Ai function. As the particle size parameter $\alpha$ increases, the surface waves exponentially die off at a faster rate; so their interference in the rainbow supernumerary region is greatly decreased, and the distortion they produce is similarly decreased.

To demonstrate the particle size effect on the $\Theta_{b}$ calculation and give a quantitative error analysis for the assumptions that are implicitly used in Airy theory, Fig. 3 gives the deviation of the fitted GO rainbow from the raytracing results for sphere radii increasing from 5 to $400 \mu \mathrm{m}$. The results for both $p=2$ and $p=3$ orders show the approach of $\left(\Theta_{\mathrm{b}}, h_{\mathrm{b}}\right)$ toward $\left(\Theta_{\mathrm{D}}, h_{\mathrm{D}}\right)$ at large particle size. Plotted in the inset of Fig. 3 is the deviation of supernumerary spacing parameter $h_{\mathrm{b}}$ from $h_{\mathrm{D}}$. For the order $p=3, h_{\mathrm{b}}$ exhibits a much slower approach toward $h_{\mathrm{D}}=27.76$ than it does toward $h_{\mathrm{D}}=4.87$ for $p=2$, which agrees with the observation that the deviation $\left(\Theta_{\mathrm{b}}-\Theta_{\mathrm{D}}\right)$ for $p=3$ is more pronounced than that for $p=2$ at the same particle size. This is because as $p$ increases, the angle of incidence of the rainbow ray increases, and fewer partial waves sample the cubic phase region; so the Debye scattering amplitude less resembles an Airy function.

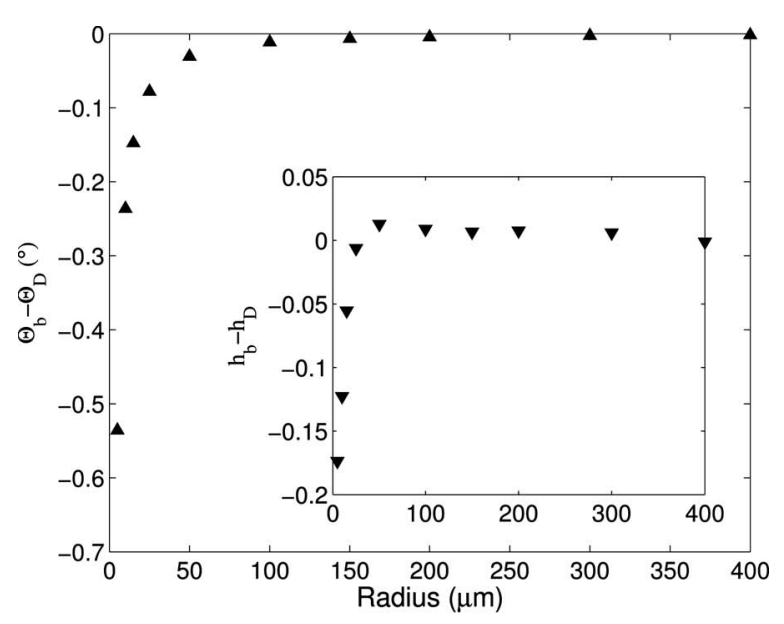

(a)

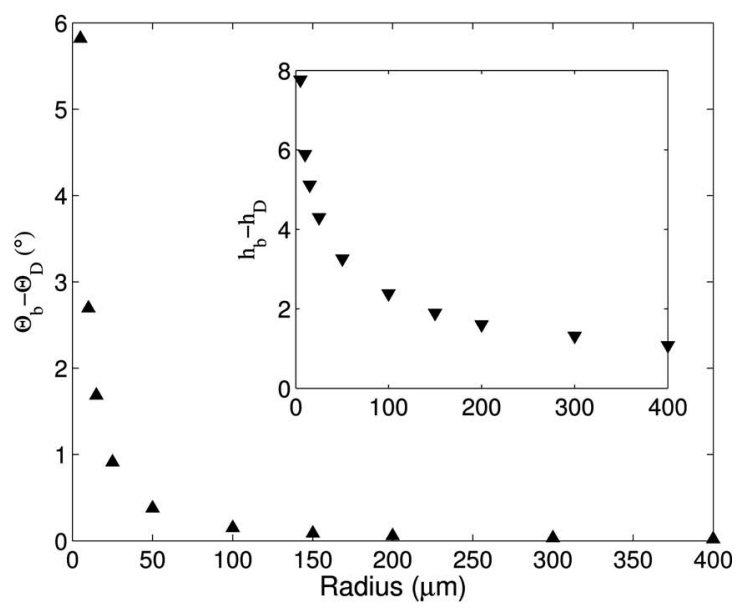

(b)

Fig. 3. Deviation of the GO rainbow angle and supernumerary spacing parameter (determined by Airy function fitting) from the results of ray tracing versus the radius of a spherical water droplet of relative refractive index $M=1.334+1.2 \mathrm{e}-9$ i. (a) $p=2$; (b) $p$ $=3$.

\section{Spheroid}

In this section, the intensity ratio law and Airy function fitting are applied to the $p=2$ intensity of a spheroid to determine the rainbow angle $\Theta_{\mathrm{b}}$. Its dependence on the spheroid eccentricity and size is evaluated. For comparison, the value of the GO rainbow $\Theta_{D}$ assumed in Airy theory is calculated by tracing a dense cluster of parallel incident rays and determining the minimum scattering angle. We parameterize the spheroid size by its volume equivalent sphere radius $r_{s}$ (hereafter called "spheroid radius") equal to $5.0 \mu \mathrm{m}$. Assume that a plane wave is polarized in the $y$ direction and incident on the spheroid along its rotationally symmetric axis (namely, end-on incidence). The TE polarized scattered wave is measured in the $O_{P}-x z$ plane $\left(\varphi=0^{\circ}\right)$, which is perpendicular to the polarization direction of the incident wave. Keeping the volume of the droplet constant but deforming it stepwise into a spheroid of axis ratios 1.05, 1.15, 1.40, and 1.50, the resultant Mie scattering intensity and its Debye series decomposition into the first five orders ( $p=0$ to 4$)$ are illustrated in Figs. 4(a), 5(a), 6(a), and 7(a), respectively. The GO decomposition of scattering at these orders is il- 
lustrated in Figs. 4(b), 5(b), 6(b), and 7(b). Comparison of the location of the rainbow intensity maximum predicted by Debye series for the order $p=2$ in Fig. 2(a) [or by GO in Fig. 2(b)] to those in Fig. 4(a) [Fig. 4(b)] and Fig. 5(a) [Fig. $5(b)]$ indicates a shift of the rainbow toward backscattering, which means a smaller impact parameter $b$ ( $b$ measures the distance of the incident ray from the rotationally symmetric axis of the spheroid) producing the rainbow. Accordingly, the angular range for two-ray interference decreases, and fewer supernumeraries are observed at the order $p=2$ as the axis ratio increases. A more straightforward linear relation between the shift of the GO rainbow $\left(\Theta_{\mathrm{b}}-\Theta_{\mathrm{D} \text {,sphere }}\right)$, and axis ratio $1.0 \leqslant a / b$ $\leqslant 1.15$ is predicted in Fig. 8 by use of both the 0.4393 law and Airy fitting of the primary bow [with the size parameter $\alpha=k_{1}(a+b) / 2$ for Eq. (49)]. Both methods predict the shift of the rainbow toward backscattering as the sphere is deformed into a prolate spheroid and in the opposite direction for an oblate spheroid. Compared with the raytracing results plotted in the same figure, the angular

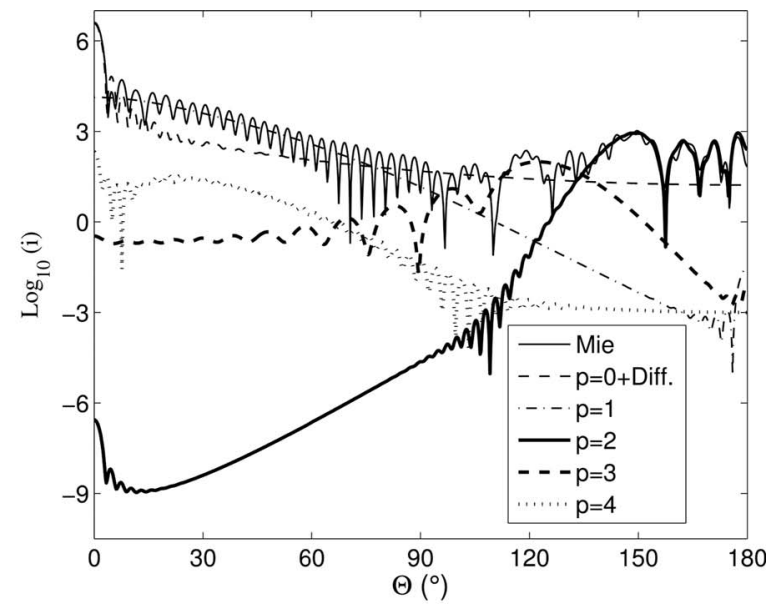

(a)

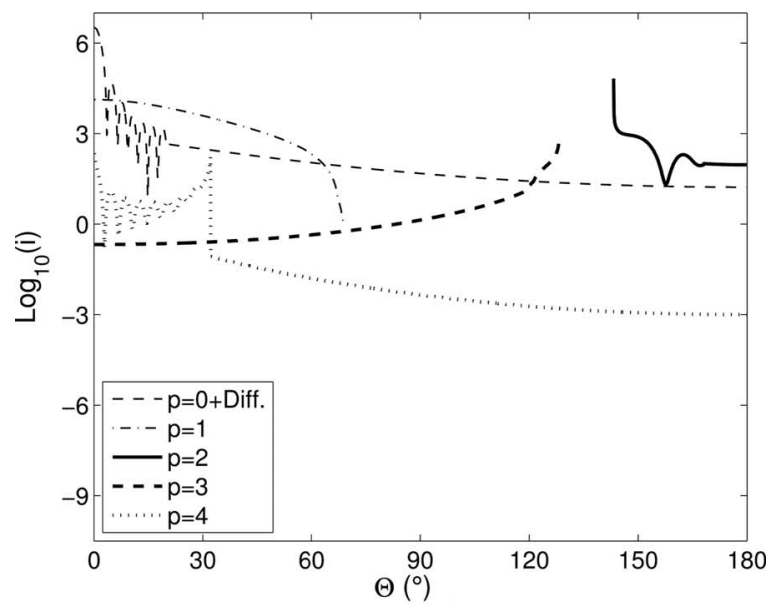

(b)

Fig. 4. (a) Mie scattering in the $\varphi=0^{\circ}$ plane and its Debye series decomposition into the first five orders $(p=0-4)$. The incident plane wave has wavelength $\lambda=0.5145 \mu \mathrm{m}$, and its electric field is polarized in the $y$ direction. The water droplet deforms from a sphere of radius $a=5.0 \mu \mathrm{m}$ into a prolate spheroid of axis ratio $a / b=1.05$. (b): GO decomposition of scattering by the same spheroid.

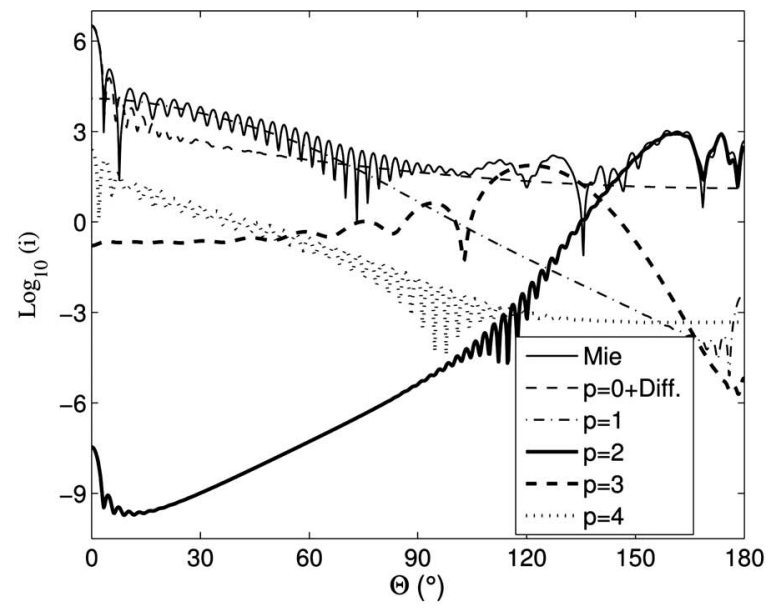

(a)

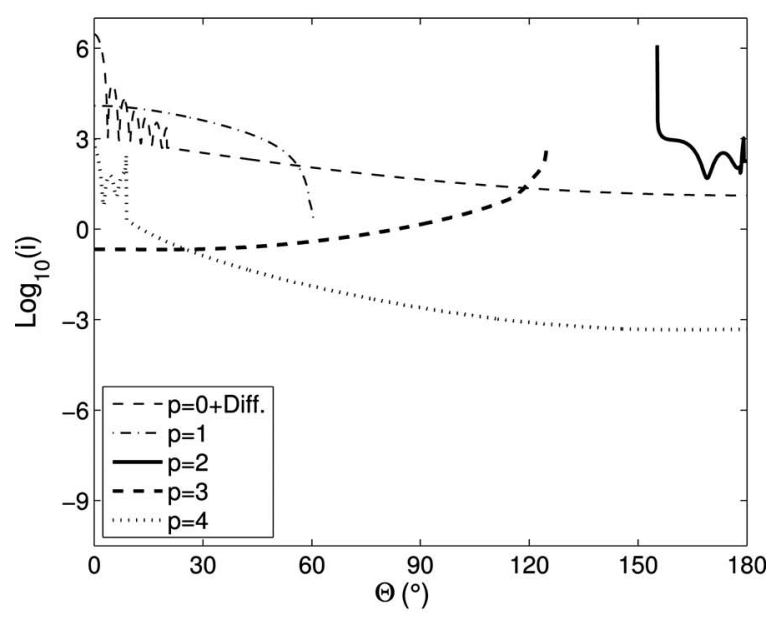

(b)

Fig. 5. (a) Same as Fig. 4(a), but the water droplet is deformed to the spheroid of axis ratio $a / b=1.15$. (b) GO decomposition of the scattering by the same spheroid.

shift of rainbow predicted by Airy function fitting for the spheroid radius as small as $5.0 \mu \mathrm{m}$ has the average deviation $0.64^{\circ}$ for prolate spheroid and $0.18^{\circ}$ for oblate spheroid. An increase of the spheroid radius to $15 \mu \mathrm{m}$ reduces the deviation to $0.22^{\circ}$ for a prolate spheroid and $0.07^{\circ}$ for an oblate spheroid (Fig. 9), which is consistent with calculations for a sphere. The improved agreement between $\Theta_{\mathrm{b}}$ and $\Theta_{\mathrm{D}}$ as well as that between $h_{\mathrm{b}}$ and $h_{\mathrm{D}}$ is more clearly demonstrated by Fig. 10 for the end-on incidence $\left(\theta_{i}=0^{\circ}\right)$ of the plane-wave on a spheroid of axis ratio $a / b=1.05$ and radius as large as $25 \mu \mathrm{m}$ (calculations are not performed for the spheroid radii larger than $25 \mu \mathrm{m}$ to avoid the numerical instabilities that occur in solving large sets of equations for the Debye series). Interestingly, Fig. 8 shows better agreement of $\Theta_{b}$ and $\Theta_{D}$ for an oblate spheroid. This is because the supernumerary spacing parameter for an oblate spheroid is smaller than that for a prolate spheroid, and, therefore, more partial waves sample the cubic phase region, so that the Airy integral has higher accuracy. This argument is supported by the observation that, for the spheroid radius $5.0 \mu \mathrm{m}$ the $p=2$ Debye intensity curve of an oblate spheroid is smoother than that of the prolate spheroid and more 


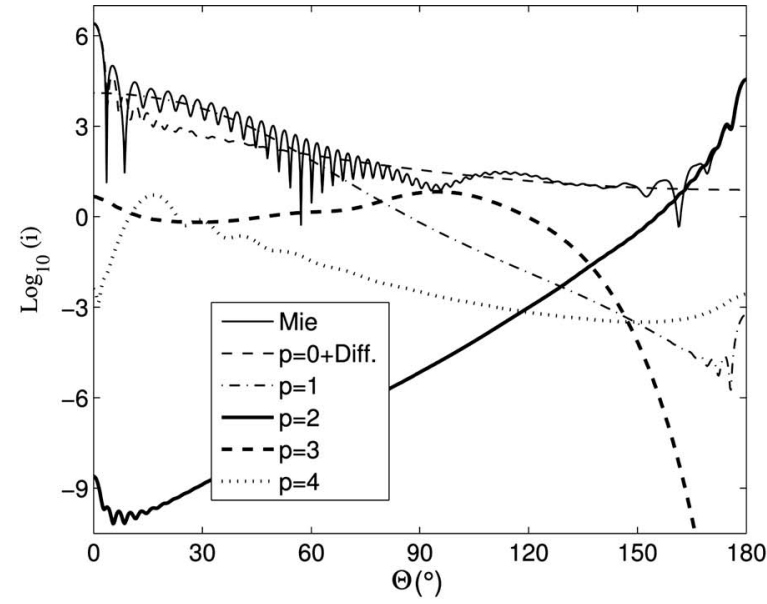

(a)

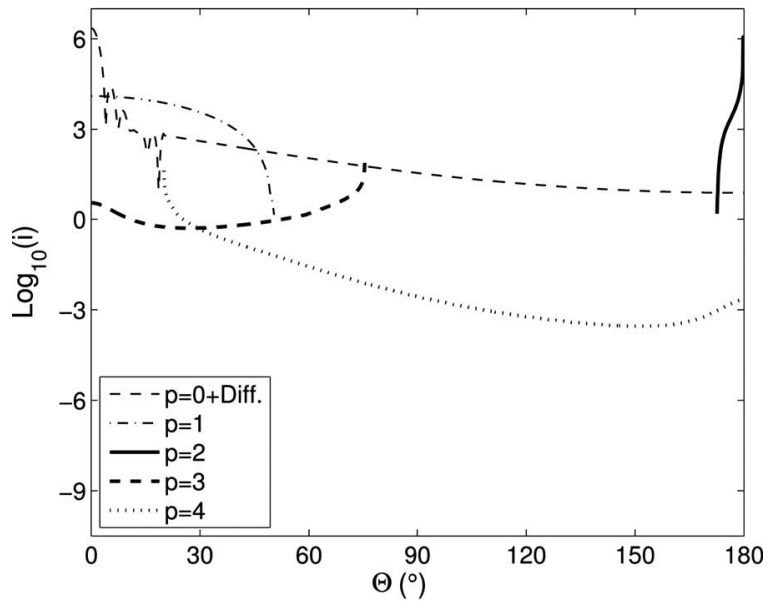

(b)

Fig. 6. Same as Fig. 4(a), but the water droplet is deformed into a spheroid of axis ratio $a / b=1.40$. (b) GO decomposition of scattering by the same spheroid.

closely resembles the Airy function shape. In addition, Fig. 8 and 9 also show the decreased accuracy of the Möbius correction [11] for large axis ratio (note that the incidence angle of the plane wave in the Möbius formula is reversed in sign from the incidence angle $\theta_{i}$ defined for the prolate spheroid in the present paper and is equal to $-\left(\pi / 2-\theta_{i}\right)$ for the oblate spheroid). The Taylor-series expansion of the rainbow angle obtained by ray tracing [12] shows that the Möbius prediction of the rainbow angle is only a first-order approximation. Such an approximation loses accuracy as the eccentricity of the spheroid increases.

As the axis ratio of the spheroid increases to $a / b$ $=1.40$, the $p=2$ order rainbow approaches the backscattering direction $\left(\Theta_{D}=179.785^{\circ}\right)$ and merges into the backward glory. The rainbow-enhanced glory causes an anomalously bright intensity distribution with $i_{\max }$ $=10^{4.548}=3.53 \mathrm{e} 4$ at $\theta=180^{\circ}$, as observed in Figs. 6(a) and $6(\mathrm{~b})$. Note that the rainbow-enhanced glory can also occur for a sphere when its refractive index is such that the rainbow shifts to the backward or forward direction [39].

For a particular axis ratio interval, the $p=2$ rays propagate through the spheroid without possessing a station-

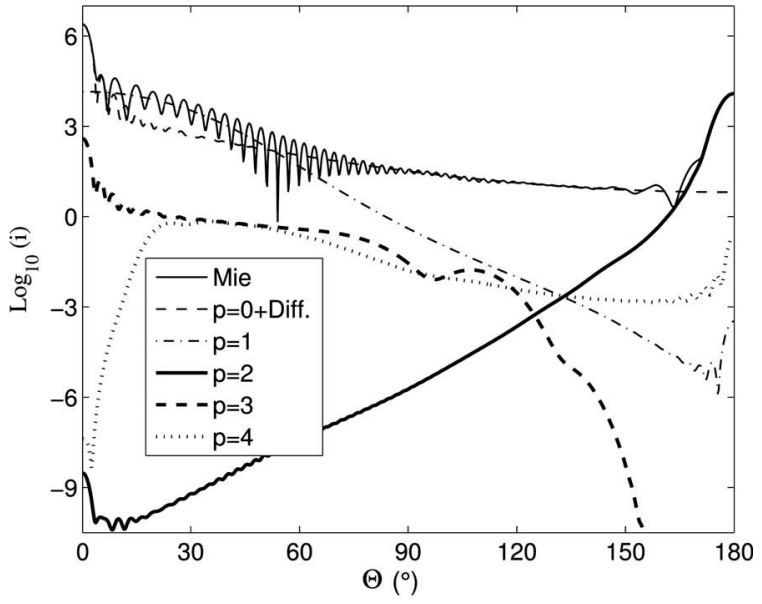

(a)

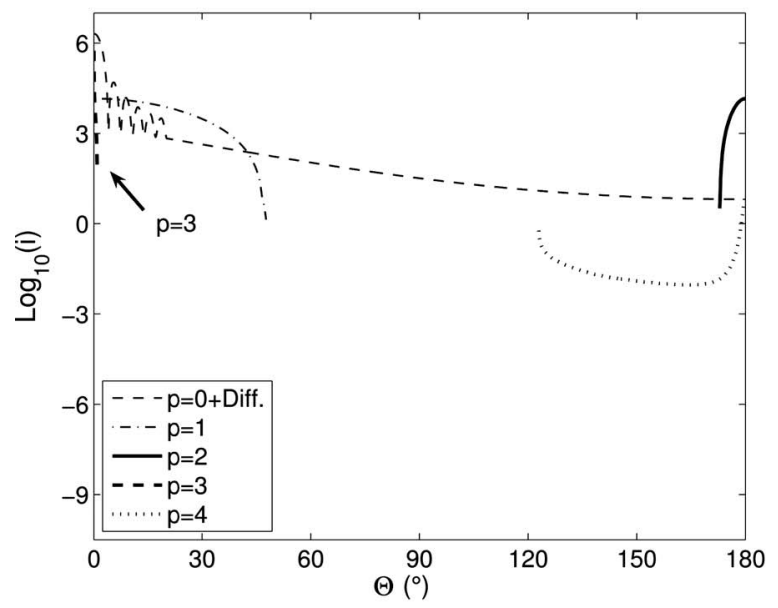

(b)

Fig. 7. Same as Fig. 4(a), but the water droplet is deformed into a spheroid of axis ratio $a / b=1.50$. (b) GO decomposition of scattering by the same spheroid.

ary deflection point leading to a rainbow. The GO analysis shows the axis ratio where the no primary-order rainbow condition begins is $(a / b)_{1}=\left[M_{r} / 2\left(M_{r}-1\right)\right]^{1 / 2}$. For this case, the paraxial rays hit the shadowed surface of the spheroid on the horizontal axis and retroreflect. Tracing the rays impacting the top hemispheroid with increasing impact parameter, the exit points decrease, have a relative minimum, and then increase. The axis ratio where the no primary-order rainbow condition ends is $(a / b)_{2}=\left[\left(2 M_{r}\right.\right.$ $\left.-1) /\left(2 M_{r}-2\right)\right]^{1 / 2}$. For this case, the paraxial rays pass through the focal point of the shadowed surface on their way both to and from the shadow surface. The exit points of the rays increase, have a relative maximum, and then decrease. But in the axis ratio interval $(a / b)_{1} \leqslant(a / b)$ $\leqslant(a / b)_{2}$, the scattering angle is a monotonically decreasing function of the impact parameter of the incident rays. This mechanism has been previously demonstrated by ray tracing [13]. For the prolate water droplet of relative refractive index $M=1.334$, the absence of the Descartes ray is predicted by GO for the axis ratio $1.4132 \leqslant a / b$ $\leqslant 1.5802$. Figure 7 gives an example for the absence of the primary rainbow for the axis ratio $a / b=1.50$. Without the participation of the rainbow, the peak value of the glory at 


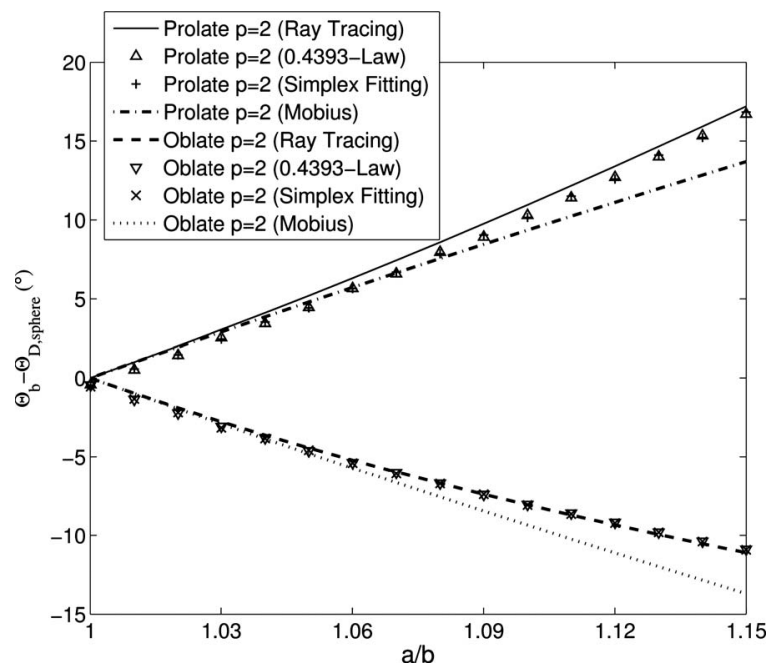

Fig. 8. Shift of the primary GO rainbow angle versus the axis ratio of prolate and oblate spheroids for $1.0 \leqslant a / b \leqslant 1.15$. The incident plane wave has wavelength $\lambda=0.5145 \mu \mathrm{m}$, and its electric field is polarized in the $y$ direction. The water droplet has the spheroid radius $r_{s}=5.0 \mu \mathrm{m}$ and relative refractive index $M$ $=1.334+1.2 \mathrm{e}-9 \mathrm{i}$. The GO rainbow angle is determined by the ray-tracing method, the 0.4393 intensity-ratio law, and the Airy function fitting by the Simplex algorithm for $p=2$ order Debye scattering in the $\varphi=0^{\circ}$ plane.

the angle $\theta=180^{\circ}$ in Fig. 7 (a) $\left(i_{\max }=10^{4.088}=1.22 \mathrm{e} 4\right)$ decreases to $\sim 1 / 3$ of the peak intensity in Fig. 6 (a) ( $i_{\max }$ $\left.=10^{4.548}=3.53 \mathrm{e} 4\right)$. Though the Descartes ray also disappears for $p=3$, an extremely narrow intensity distribution in the near-forward direction is observed in the Debye intensity curve of Fig. 7(a). This is due to the forward focusing of the emergent rays after two internal reflections, which is verified by computer ray tracing. As an exception to the general agreement between the angular rainbow location predicted by Debye series and GO, the GO rainbow at $122.9^{\circ}$ for $p=4$ [see Fig. $7(\mathrm{~b})$ ] is not observed at the same scattering angle in the Debye curve. Instead, the $p$ $=4$ order rainbow maximum shifts far forward to the angle $\sim 35^{\circ}$. The computer ray tracing shows a much higher sensitivity of the $p=4$ order GO rainbow on the eccentricity than that of the lower-order rainbows. It shifts

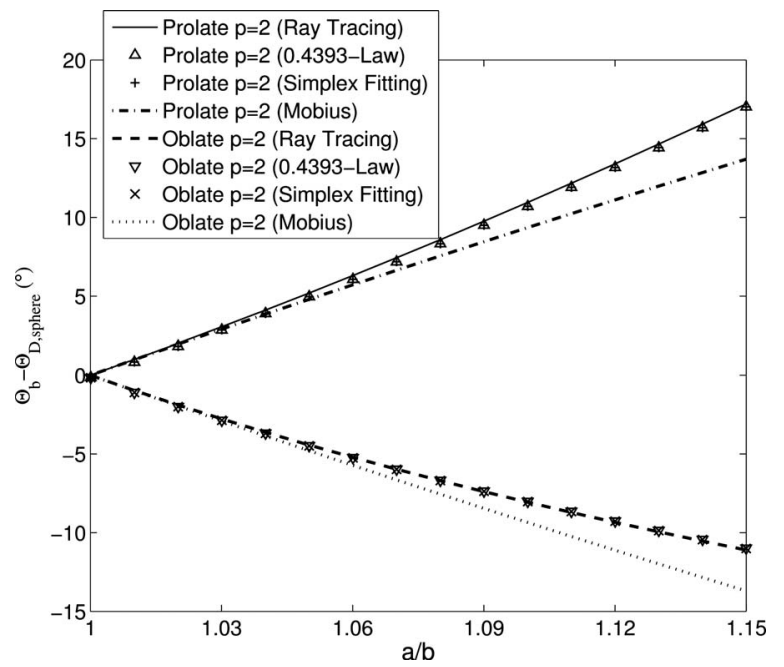

Fig. 9. Same as in Fig. 8, but the spheroid radius is $15.0 \mu \mathrm{m}$.

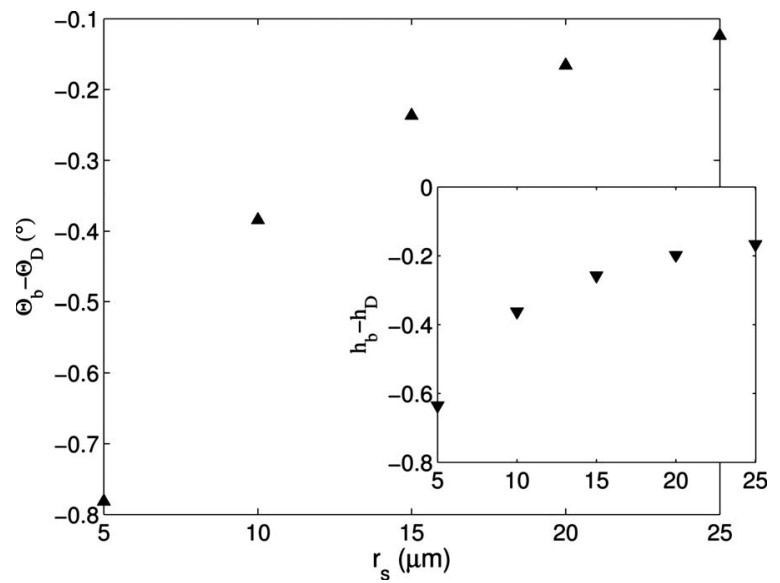

Fig. 10. Deviation of the primary GO rainbow angle and supernumerary spacing parameter (determined by Airy function fitting) from the ray-tracing results for the spheroid radius increasing from 5.0 to $25.0 \mu \mathrm{m}$. The spheroid has the axis ratio $a / b$ $=1.05$ and is illuminated by an end-on incident plane-wave $\left(\theta_{i}\right.$ $\left.=0^{\circ}\right)$. Both $\Theta_{\mathrm{D}}$ and $h_{\mathrm{D}}$ are obtained for the spheroid by tracing a dense cluster of parallel incident rays.

from $\Theta_{\mathrm{D}}=90^{\circ}$ to $\Theta_{\mathrm{D}}=180^{\circ}$ as $a / b$ slightly increases from 1.4935 to 1.5092 . In wave theory, however, at the spheroid radius of $5.0 \mu \mathrm{m}$ the dramatic response of the $p=4$ order rainbow location to a slight eccentricity variation is likely obscured by surface waves that have a stronger contribution to the scattering around the rainbow than the real rays and extend the scattering to more forward angles.

\section{B. Rainbow for Oblique Wave Incidence}

Unlike the invariant location of the rainbow for a sphere with respect to the propagation direction of the incident plane wave, the rainbow for a spheroid changes its angular location, since the spheroid is a body of lower symmetry. In this subsection a wave theory analysis is made for prolate spheroids of radius $r_{s}=8.0 \mu \mathrm{m}$ and axis ratios $1.01,1.03$, and 1.06. We vary the propagation direction of the plane wave from end-on incidence $\left(\theta_{i}=0^{\circ}\right)$ to side-on incidence $\left(\theta_{i}=90^{\circ}\right)$ with the step of incidence angle $\Delta \theta_{i}$ $=10^{\circ}$, but keeping the polarization of the electric field along the $y$ axis $\left(\varphi_{i}=90^{\circ}\right)$ so that the scattering in the $O_{P}-x y$ plane $\left(\varphi=0^{\circ}\right)$ is dominated by the TE wave (the contribution to the $\varphi=0^{\circ}$ scattering plane by the rays with incidence planes $\varphi \neq 0^{\circ}$ is assumed to be negligible). Comparison of the GO rainbows determined by the 0.4393 law and Airy fitting to the ray-tracing determined rainbow for a spheroid is illustrated in Figs. 11(a)-11(c). A general sinusoidal-shaped curve of the rainbow deviation from its average value $\Theta_{\mathrm{b} \text {,ave }}$ versus the incidence angle $\theta_{i}$ is predicted by all methods. The Airy-fitting determined rainbow $\Theta_{\mathrm{b}}$ for $a / b=1.01,1.03,1.06$ differs from the raytracing result by $0.29^{\circ}, 0.28^{\circ}$, and $0.24^{\circ}$, respectively. Again, it is observed in Fig. 11 that the deviation of Möbius-predicted rainbow from the ray-tracing result increases as the eccentricity increases.

In the cubic shape approximation of the wavefront of the rays leaving the elliptical cross section of the spheroid around the rainbow ray, the supernumerary spacing parameter describes the angular spacing of the maxima and minima of the rainbow structure. Although an analytical 


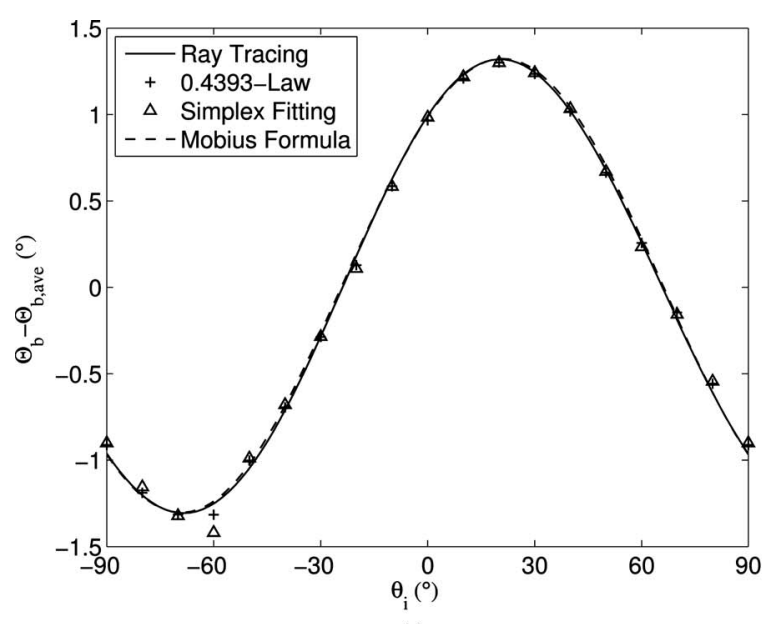

(a)

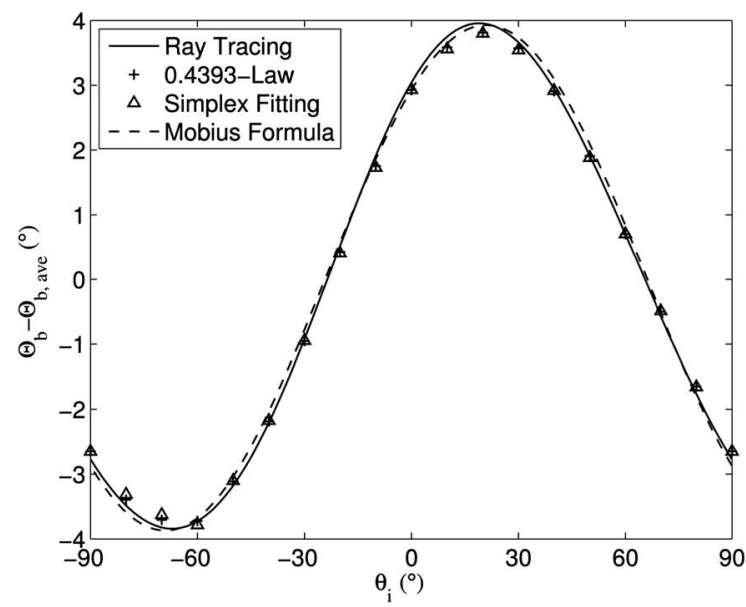

(b)

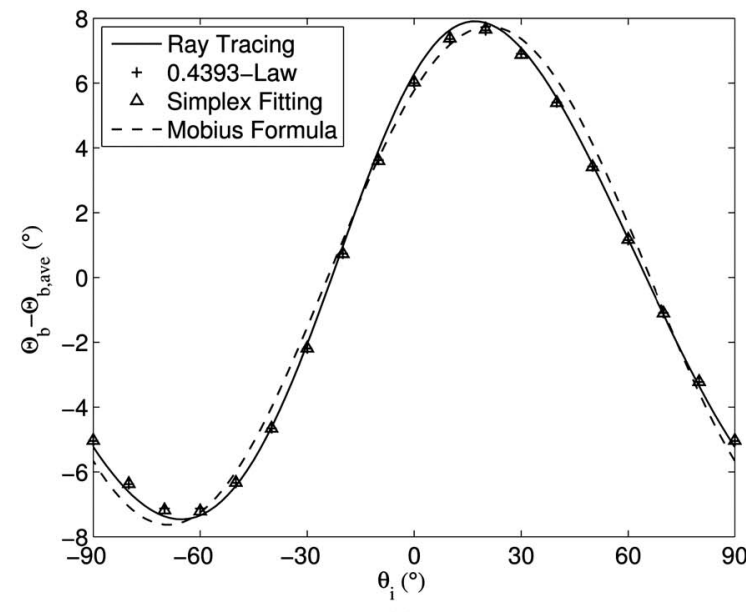

(c)

Fig. 11. Deviation of the primary GO rainbow angle $\Theta_{b}$ from its average value $\Theta_{b \text {,ave }}$ versus the incidence angle of the plane wave on a prolate spheroid of relative refractive index $M=1.334$ $+1.2 \mathrm{e}-9 \mathrm{i}$, the spheroid radius $r_{s}=8.0 \mu \mathrm{m}$, and axis ratio is (a) 1.01 , (b) 1.03 , and (c) 1.06 . The droplet is illuminated by a planewave of wavelength $\lambda=0.5145 \mu \mathrm{m}$ with incidence angle ranging from $0^{\circ}$ to $90^{\circ}$ and the electric field polarized in the $y$ direction. The rainbow in the scattering plane $\varphi=0^{\circ}$ is calculated by the ray-tracing method, the 0.4393 intensity-ratio law, Airy function fitting by the Simplex algorithm, and the Möbius formula. expression for $h_{\mathrm{D}}$ is difficult to obtain, a numerical solution is available via calculating the optical path length of the incident rays from the entrance plane to the exit plane, multiplying it by the wave number to get the phase of the rays around the rainbow ray, and determining $h_{\mathrm{D}}$ as the coefficient multiplying the cubic term in series expansion of the phase [34]. As compared in Fig. 12, both Airy fitting and ray tracing yield an approximately sinusoidal-shaped curve of $h$. By using a Fourier expansion for $h$, the discrepancy between $h_{\mathrm{b}}$ and $h_{\mathrm{D}}$ is found to be caused mainly by the constant term, which is independent of the incidence angle but is dependent on the spheroid axis ratio and spheroid size.

\section{CONCLUSION}

Spheroid scattering can be analyzed by the Debye series developed in the present paper. As an example, the dependence of rainbow scattering on the spheroid eccentricity is studied. Two Airy-function-based methods including the intensity-ratio law and Airy fitting are used to derive the first-order GO rainbow angle and supernumerary spacing parameter from the $p=2$ order Debye scattering. The implicit assumption of the standard Airy correction that the Descartes rainbow location and the supernumerary spacing parameter are given by ray-tracing and Airy theory, respectively, are tested and the particle size effect is analyzed. Numerical calculations support the following predictions of GO:

1. For end-on plane-wave incidence the rainbow has a linear shift toward larger scattering angles as the sphere deforms into the prolate spheroid with axis ratio less than 1.15. The direction of the shift reverses if the deformation is into an oblate spheroid.

2. For end-on plane-wave incidence, the rainbow disappears when the spheroid eccentricity is in the interval $\left[M_{r} / 2\left(M_{r}-1\right)\right]^{1 / 2} \leqslant a / b \leqslant\left[\left(2 M_{r}-1\right) /\left(2 M_{r}-2\right)\right]^{1 / 2}$ owing to the lack of stationary deflection of the emergent rays.

3. A rainbow-enhanced glory is produced at the axis ratios for which the rainbow ray leaves the particle in the backward or forward direction.

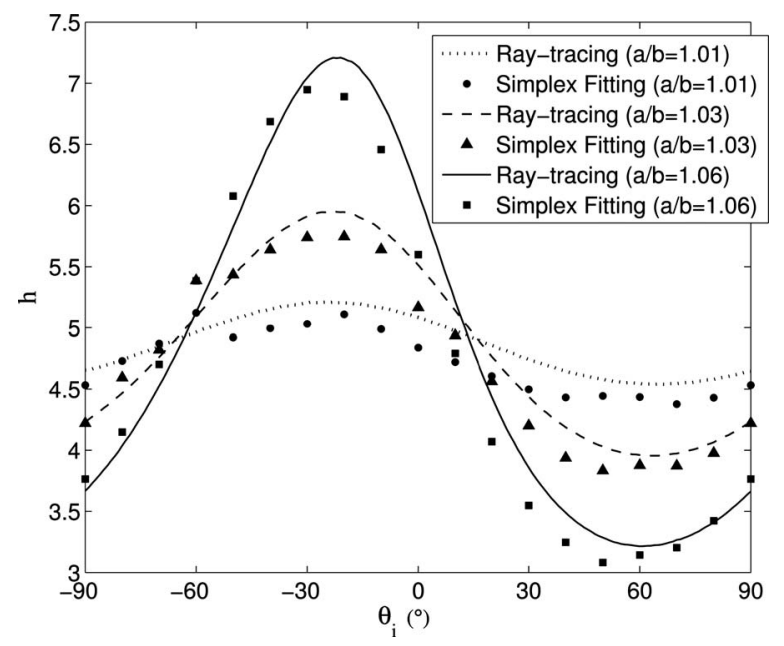

Fig. 12. The primary supernumerary spacing parameter determined by Airy function fitting. The incident wave and the particle are the same as in Fig. 11. 
4. The rainbow and supernumerary spacing parameter show sinusoidal shapes with respect to the incidence angle of the incoming wave for a spheroid of small eccentricity.

We also show that, owing to the contribution of more partial waves as the particle size increases, the values of GO rainbow angle and the supernumerary spacing parameter assumed in Airy theory become more accurate in describing the rainbow scattering.

However, it is noteworthy that in all our demonstration calculations for oblique wave incidence on a spheroid of axis ratio $a / b \leqslant 1.06$, we assume rainbow scattering to be dominated by the TE polarization in the scattering plane of $\varphi=0^{\circ}$. However, for a larger axis ratio the TE rainbow might interfere with the rays leaving the droplet in the same direction but with the incidence planes other than the $\varphi=0^{\circ}$ plane [40]. The interference might bias the TE wave signals and cause cross polarization of the scattered light. Analysis of the rainbow in this case needs further study, as done for horizontal rainbow scattering of a side-on incident beam by an oblate droplet [18].

\section{APPENDIX A}

For scattering of an incident beam by a spheroid, the derivation of the Debye series decomposition of the scattering amplitudes differs from that for scattering by a sphere or a circular cylinder because all the scattering amplitudes involved are now matrices rather than scalars. In order to illustrate our method, we first outline the derivation of the Debye series for scattering of scalar waves by a spheroid. We then describe the additional ingredients required for the derivation for scattering of electromagnetic waves.

When the scalar wave equation is separated in spheroidal coordinates and a wave with wave number $k$ is decomposed in terms of an infinite series of partial waves $n$ and azimuthal modes $m$, the wave amplitude $\psi$ takes the form

$$
\psi(\xi, \eta, \varphi)=\sum_{m=-\infty}^{\infty} \sum_{n=|m|, n \neq 0}^{\infty} X_{n}^{m} R_{|m| n}^{(j)}(c ; \xi) S_{|m| n}(c ; \eta) \exp (\operatorname{im} \varphi)
$$

where the spheroidal coordinates are $(\xi, \mu, \varphi)$, the radial spheroidal function is $R_{m n}^{(j)}$ with $j=1,2,3,4$, the angular spheroidal function is $S_{m m}, c$ is related to the spheroidal coordinate system focal length $f$ by $c=k f$, and $X_{n}^{m}$ are constants. The derivation of the Debye series decomposition of the partial wave amplitudes for scattering of scalar waves by a spheroid consists of five steps.

Step 1. Consider a radially incoming wave with partial wave coefficients $(1 / 2) G_{n}^{m}$ in an exterior medium (medium 2) of refractive index $M_{2}$, which is incident on the surface of a spheroid of size parameter $\xi_{0}$ and refractive index $M_{1}$ (medium 1). The interaction of the incident wave with the spheroid surface creates both a radially outgoing reflected wave in the external medium with partial wave amplitudes $R_{n}^{m, 22}$ and a radially incoming transmitted wave with amplitudes $T_{n}^{m, 21}$. The incident, reflected, and interior waves are

$$
\psi_{\mathrm{inc}}=\frac{1}{2} \sum_{m=-\infty}^{\infty} \sum_{n=|m|, n \neq 0}^{\infty} G_{n}^{m} R_{|m| n}^{(4)}\left(c_{2} ; \xi\right) S_{|m| n}\left(c_{2} ; \eta\right) \exp (i m \varphi)
$$

$$
\psi_{\mathrm{ref}}=\sum_{m=-\infty}^{\infty} \sum_{n=|m|, n \neq 0}^{\infty} R_{n}^{m, 22} R_{|m| n}^{(3)}\left(c_{2} ; \xi\right) S_{|m| n}\left(c_{2} ; \eta\right) \exp (\operatorname{im} \varphi)
$$

$$
\psi_{\text {trans }}=\sum_{m=-\infty}^{\infty} \sum_{n=|m|, n \neq 0}^{\infty} T_{n}^{m, 21} R_{|m| n}^{(4)}\left(c_{1} ; \xi\right) S_{|m| n}\left(c_{1} ; \eta\right) \exp (i m \varphi)
$$

where $c_{1}=k_{1} f$ and $c_{2}=M_{2} c_{1} / M_{1}=k_{2} f$. Continuity of both the wave and its normal derivative at $\xi=\xi_{0}$ permits the evaluation of $R_{n}^{m, 22}$ and $T_{n}^{m, 21}$ in terms of $G_{n}^{m}$. The results take the form

$$
\begin{aligned}
& R_{n}^{m, 22}=\frac{1}{2} \sum_{n_{1}=|m|, n_{1} \neq 0}^{\infty} R_{n, n_{1}}^{m, 22} G_{n_{1}}^{m}, \\
& T_{n}^{m, 21}=\frac{1}{2} \sum_{n_{1}=|m|, n_{1} \neq 0}^{\infty} T_{n, n_{1}}^{m, 21} G_{n_{1}}^{m} .
\end{aligned}
$$

The incoming partial wave $\left(m, n_{1}\right)$ produces a reflected and transmitted contribution for all other partial waves $(m, n)$. For scattering of a plane wave by a sphere or circular cylinder, this partial wave coupling did not occur. The incident partial wave $\left(m, n_{1}\right)$ in those cases coupled only to the $\left(m, n_{1}\right)$ interior and scattered partial waves. In general, off-diagonal partial wave coupling occurs when the scattering particle has lower symmetry that of a sphere or circular cylinder. Thus the transmitted and reflected amplitudes of Eqs. (A5) and (A6) are matrices in the indices $n$ and $n_{1}$ and reduce to diagonal matrices in the sphere limit.

Step 2. Consider now a radially outgoing wave with partial wave coefficients $(1 / 2) J_{n}^{m}$ inside the spheroid and incident on its surface. The interaction of the incident wave with the spheroid surface creates both a radially incoming reflected wave inside the spheroid with partial wave amplitudes $R_{n}^{m, 11}$ and a radially outgoing transmitted wave in the exterior medium with amplitudes $T_{n}^{m, 12}$. The incident, reflected, and interior waves are

$$
\begin{aligned}
& \psi_{\text {inc }}=\frac{1}{2} \sum_{m=-\infty}^{\infty} \sum_{n=|m|, n \neq 0}^{\infty} J_{n}^{m} R_{|m| n}^{(3)}\left(c_{1} ; \xi\right) S_{|m| n}\left(c_{1} ; \eta\right) \exp (i m \varphi) \\
& \psi_{\text {ref }}=\sum_{m=-\infty}^{\infty} \sum_{n=|m|, n \neq 0}^{\infty} R_{n}^{m, 11} R_{|m| n}^{(4)}\left(c_{1} ; \xi\right) S_{|m| n}\left(c_{1} ; \eta\right) \exp (\operatorname{im} \varphi),
\end{aligned}
$$




$$
\psi_{\text {trans }}=\sum_{m=-\infty}^{\infty} \sum_{n=|m|, n \neq 0}^{\infty} T_{n}^{m, 12} R_{|m| n}^{(3)}\left(c_{2} ; \xi\right) S_{|m| n}\left(c_{2} ; \eta\right) \exp (i m \varphi) .
$$

Continuity of both the wave and its normal derivative at $\xi=\xi_{0}$ again permits the evaluation of $R_{n}^{m, 11}$ and $T_{n}^{m, 12}$ in terms of $J_{n}^{m}$. The results again are of the form

$$
\begin{aligned}
R_{n}^{m, 11} & =\frac{1}{2} \sum_{n_{1}=|m|, n_{1} \neq 0}^{\infty} R_{n, n_{1}}^{m, 11} J_{n_{1}}^{m}, \\
T_{n}^{m, 12} & =\frac{1}{2} \sum_{n_{1}=|m|, n_{1} \neq 0}^{\infty} T_{n, n_{1}}^{m, 12} J_{n_{1}}^{m},
\end{aligned}
$$

again illustrating partial wave coupling between the incident partial wave $n_{1}$ and the reflected and transmitted partial waves $n$.

Step 3. Now consider the full Mie scattering problem of an incident plane wave or focused beam incident on the spheroid. In this case the incident, scattered, and interior waves take the form

$$
\psi_{\text {inc }}=\sum_{m=-\infty}^{\infty} \sum_{n=|m|, n \neq 0}^{\infty} G_{n}^{m} R_{|m| n}^{(1)}\left(c_{2} ; \xi\right) S_{|m| n}\left(c_{2} ; \eta\right) \exp (\operatorname{im} \varphi),
$$

$$
\psi_{\mathrm{sca}}=-\sum_{m=-\infty}^{\infty} \sum_{n=|m|, n \neq 0}^{\infty} B_{n}^{m} R_{|m| n}^{(3)}\left(c_{2} ; \xi\right) S_{|m| n}\left(c_{2} ; \eta\right) \exp (i m \varphi),
$$

$$
\psi_{\mathrm{int}}=\sum_{m=-\infty}^{\infty} \sum_{n=|m|, n \neq 0}^{\infty} D_{n}^{m} R_{|m| n}^{(1)}\left(c_{1} ; \xi\right) S_{|m| n}\left(c_{1} ; \eta\right) \exp (i m \varphi),
$$

where $B_{n}^{m}$ are the partial wave scattering amplitudes and $D_{n}^{m}$ are the partial wave interior amplitudes. Again these amplitudes are of the form

$$
\begin{aligned}
& B_{n}^{m}=\sum_{n_{1}=|m|, n_{1} \neq 0}^{\infty} B_{n, n_{1}}^{m} G_{n_{1}}^{m}, \\
& D_{n}^{m}=\sum_{n_{1}=|m|, n_{1} \neq 0}^{\infty} D_{n, n_{1}}^{m} G_{n_{1}}^{m} .
\end{aligned}
$$

Step 4. In order to evaluate $D_{n, n_{1}}^{m}$ in terms of the partial wave reflection and transmission amplitudes, the interior field of Eq. (A4) is added to the total interior field of Eqs. (A7) and (A8). The result is then cast in the form of a standing wave $R_{m n}^{(1)}\left(c_{1} ; \xi\right)$ in accordance with Eq. (A14). This is possible only when

$$
D_{n}^{m}=\sum_{n_{1}=|m|, n_{1} \neq 0}^{\infty} T_{n, n_{1}}^{m, 21} G_{n_{1}}^{m}+R_{n, n_{1}}^{m, 11} J_{n_{1}}^{m},
$$

$$
\begin{aligned}
J_{n}^{m}= & \sum_{n_{1}=|m|, n_{1} \neq 0}^{\infty} \sum_{n_{2}=|m|, n_{2} \neq 0}^{\infty}(\boldsymbol{I} \\
& \left.-R^{m, 11}\right)_{n, n_{1}}^{-1} T_{n_{1}, n_{2}}^{m, 21} G_{n_{2}}^{m},
\end{aligned}
$$

where $\boldsymbol{I}$ is the identity matrix. The interior partial wave amplitudes are then

$$
D_{n, n_{1}}^{m}=\sum_{n_{2}=|m|, n_{2} \neq 0}^{\infty}\left(\boldsymbol{I}-R^{m, 11}\right)_{n, n_{2}}^{-1} T_{n_{2}, n_{1}}^{m, 21} .
$$

The interior Debye series is given by Eq. (A19) by using the identity

$$
\begin{aligned}
\left(\boldsymbol{I}-R^{m, 11}\right)_{n, n_{2}}^{-1}= & \delta_{n, n_{2}}+R_{n, n_{2}}^{m, 11}+\sum_{n_{3}=|m|, n_{3} \neq 0}^{\infty} R_{n, n_{3}}^{m, 11} R_{n_{3}, n_{2}}^{m, 11} \\
& +\sum_{n_{4}=|m|, n_{4} \neq 0}^{\infty} \sum_{n_{3}=|m|, n_{3} \neq 0}^{\infty} R_{n, n_{4}}^{m, 11} R_{n_{4}, n_{3}}^{m, 11} R_{n_{3}, n_{2}}^{m, 11} \\
& +\ldots
\end{aligned}
$$

Step 5. In order to evaluate $B_{n, n_{1}}^{m}$ in terms of the partial wave reflection and transmission amplitudes, one now adds the total exterior field of Eqs. (A2) and (A3) to the exterior field of Eq. (A9) and demands that the result is the combination of a standing wave of the form $R_{|m| n}^{(1)}\left(c_{2} ; \xi\right)$ plus an outgoing wave of the form $R_{|m| n}^{(3)}\left(c_{2} ; \xi\right)$ in accordance with Eqs. (A12) and (A13). This gives

$$
B_{n}^{m}=\frac{1}{2} \sum_{n_{1}=|m|, n_{1} \neq 0}^{\infty}\left(\delta_{n, n_{1}} G_{n_{1}}^{m}-R_{n, n_{1}}^{m, 22} G_{n_{1}}^{m}-T_{n, n_{1}}^{m, 12} J_{n_{1}}^{m}\right) .
$$

Substituting Eq. (A17) into Eq. (A21), one obtains

$$
\begin{aligned}
B_{n, n_{1}}^{m}= & \frac{1}{2}\left[\delta_{n, n_{1}}-R_{n, n_{1}}^{m, 22}-\sum_{n_{2}=|m|, n_{2} \neq 0}^{\infty} \sum_{n_{3}=|m|, n_{3} \neq 0}^{\infty} T_{n, n_{2}}^{m, 12}(\boldsymbol{I}\right. \\
& \left.\left.-R^{m, 11}\right)_{n_{2}, n_{3}}^{-1} T_{n_{3}, n_{1}}^{m, 21}\right] .
\end{aligned}
$$

Equations (A20) and (A22) are the Debye series decomposition of the spheroid partial wave scattering amplitudes for incident scalar waves.

The derivation of the Debye series for scattering of an electromagnetic wave by a spheroid is obtained by using the same general method, with the following additions. As described in Section 2, the wave amplitude $\psi$ of Eq. (A1) is replaced by the electric and magnetic fields of the incident, transmitted, reflected, interior, and scattered waves. Each of these fields also has a TE component and a TM component with its own TE and TM partial wave coefficient. The TM and TE partial wave scattering amplitudes are $A_{n}^{m}$ and $B_{n}^{m}$, and the TM and TE partial wave interior amplitudes are $C_{n}^{m}$ and $D_{n}^{m}$. As was the case for scattering of a plane wave with diagonal incidence by a circular cylinder, at each interaction of an incident partial wave with the spheroid surface both polarizationpreserving waves (i.e., TE to TE and TM to TM) and cross-polarized waves (i.e., TE to TM and TM to TE) are created. We call these four polarization-channel ampli- 
tudes TE/TE, TM/TM, TM/TE and TE/TM, respectively. Thus in steps 1 and 2 of the derivation for scalar waves, Eqs. (A5) and (A6), for example, are replaced by

$$
\begin{aligned}
R_{n}^{m, 22, T E}= & \frac{1}{2} \sum_{n_{1}=|m|, n_{1} \neq 0}^{\infty}\left(R_{n, n_{1}}^{m, 22, T E / T E} G_{n_{1}}^{m, T E}\right. \\
& \left.+R_{n, n_{1}}^{m, 22, T E / T M} G_{n_{1}}^{m, T M}\right), \\
R_{n}^{m, 22, T M}= & \frac{1}{2} \sum_{n_{1}=|m|, n_{1} \neq 0}^{\infty}\left(R_{n, n_{1}}^{m, 22, T M / T E} G_{n_{1}}^{m, T E}\right. \\
& \left.+R_{n, n_{1}}^{m, 22, T M / T M} G_{n_{1}}^{m, T M}\right), \\
T_{n}^{m, 21, T E}= & \frac{1}{2} \sum_{n_{1}=|m|, n_{1} \neq 0}^{\infty}\left(T_{n, n_{1}}^{m, 21, T E / T E} G_{n_{1}}^{m, T E}\right. \\
& \left.+T_{n, n_{1}}^{m, 21, T E / T M} G_{n_{1}}^{m, T M}\right), \\
T_{n}^{m, 21, T M}= & \frac{1}{2} \sum_{n_{1}=|m|, n_{1} \neq 0}^{\infty}\left(T_{n, n_{1}}^{m, 21, T M / T E} G_{n_{1}}^{m, T E}\right. \\
& \left.+T_{n, n_{1}}^{m, 21, T M / T M} G_{n_{1}}^{m, T M}\right) .
\end{aligned}
$$

Similarly, the partial wave interior amplitudes are

$$
\begin{aligned}
C_{n}^{m}= & \sum_{n_{1}=|m|, n_{1} \neq 0}^{\infty}\left(R_{n, n_{1}}^{m, 11, T M / T E} J_{n_{1}}^{m, T E}+R_{n, n_{1}}^{m, 11, T M / T M} J_{n_{1}}^{m, T M}\right. \\
& \left.+T_{n, n_{1}}^{m, 21, T M / T E} G_{n_{1}}^{m, T E}+T_{n, n_{1}}^{m, 21, T M / T M} G_{n_{1}}^{m, T M}\right), \\
D_{n}^{m}= & \sum_{n_{1}=|m|, n_{1} \neq 0}^{\infty}\left(R_{n, n_{1}}^{m, 11, T E / T M} J_{n_{1}}^{m, T M}+R_{n, n_{1}}^{m, 11, T E / T E} J_{n_{1}}^{m, T E}\right. \\
& \left.+T_{n, n_{1}}^{m, 21, T E / T M} G_{n_{1}}^{m, T M}+T_{n, n_{1}}^{m, 21, T E / T E} G_{n_{1}}^{m, T E}\right),
\end{aligned}
$$

and the partial wave scattering amplitudes are

$$
\begin{aligned}
A_{n}^{m}= & \frac{1}{2} G_{n}^{m, T M}-\frac{1}{2} \sum_{n_{1}=|m|, n_{1} \neq 0}^{\infty}\left(R_{n, n_{1}}^{m, T 2, T M / T E} G_{n_{1}}^{m, T E}\right. \\
& +R_{n, n_{1}}^{m, 22, T M / T M} G_{n_{1}}^{m, T M}+T_{n, n_{1}}^{m, 12, T M / T E} J_{n_{1}}^{m, T E} \\
& \left.+T_{n, n_{1}}^{m, 12, T M / T M} J_{n_{1}}^{m, T M}\right), \\
B_{n}^{m}= & \frac{1}{2} G_{n}^{m, T E}-\frac{1}{2} \sum_{n_{1}=|m|, n_{1} \neq 0}^{\infty}\left(R_{n, n_{1}}^{m, 22, T E / T M} G_{n_{1}}^{m, T M}\right. \\
& +R_{n, n_{1}}^{m, 22, T E / T E} G_{n_{1}}^{m, T E}+T_{n, n_{1}}^{m, 12, T E / T M} J_{n_{1}}^{m, T M} \\
& \left.+T_{n, n_{1}}^{m, 12, T E / T E} J_{n_{1}}^{m, T E}\right) .
\end{aligned}
$$

The reflection, transmission, interior, and scattering amplitudes are not only matrices in the indices $n_{1}$ and $n$, but also in the polarization states TE and TM as well.

Once Eqs. (A23), (A25), and (A26) and the expressions analogous to Eqs. (A10) and (A11) that depend on $J_{n_{1}}^{m, T E}$ and $J_{n_{1}}^{m, T M}$ are substituted into the equations for the interior electric and magnetic fields, the result is a type- 1 radial standing wave only if the incident amplitudes $J_{n_{1}}^{m, T E}$ and $J_{n_{1}}^{m, T M}$ are related to the incident amplitudes $G_{n_{1}}^{m, T E}$ and $G_{n_{1}}^{n, T M}$ by the sets of simultaneous equations

$$
\begin{aligned}
J_{n_{2}}^{m, T E}= & \sum_{n=|m|, n \neq 0}^{\infty} \sum_{n_{1}=|m|, n_{1} \neq 0}^{\infty}[(\boldsymbol{I} \\
& \left.-R^{m, 11, T E / T E}\right)_{n_{2}, n}^{-1} R_{n, n_{1}}^{m, 11, T E / T M} J_{n_{1}}^{m, T M}+(\boldsymbol{I} \\
& \left.-R^{m, 11, T E / T E}\right)_{n_{2}, n}^{-1} T_{n, n_{1}}^{m, 21, T E / T M} G_{n_{1}}^{m, T M}+(\boldsymbol{I} \\
& \left.\left.-R^{m, 11, T E / T E}\right)_{n_{2}, n}^{-1} T_{n, n_{1}}^{m, 21, T E / T E} G_{n_{1}}^{m, T E}\right], \\
J_{n_{2}}^{m, T M}= & \sum_{n=|m|, n \neq 0}^{\infty} \sum_{n_{1}=|m|, n_{1} \neq 0}^{\infty}[(\boldsymbol{I} \\
& \left.-R^{m, 11, T M / T M}\right)_{n_{2}, n}^{-1} R_{n, n_{1}}^{m, 11, T M / T E} J_{n_{1}}^{m, T E}+(\boldsymbol{I} \\
& \left.-R^{m, 11, T M / T M}\right)_{n_{2}, n}^{-1} T_{n, n_{1}}^{m, 21, T M / T E} G_{n_{1}}^{m, T E}+(\boldsymbol{I} \\
& \left.\left.-R^{m, 11, T M / T M}\right)_{n_{2}, n}^{-1} T_{n, n_{1}}^{m, 21, T M / T M} G_{n_{1}}^{m, T M}\right] .
\end{aligned}
$$

Iterating these expressions for $J_{n_{2}}^{m, T E}$ and $J_{n_{2}}^{m, T M}$ and substituting the results into the interior fields of Eqs. (A27) and (A28) then gives the interior Debye series, and substituting them into the exterior fields of Eqs. (A29) and (A30) gives the Debye series for the partial wave scattering amplitudes. The results of this procedure iterated to any given number of interactions of the incident waves with the surface of the spheroid are given in Section 2.

\section{ACKNOWLEDGMENTS}

This research is supported by the German Research Foundation (grant TR194/49-1) and the Alexander von Humboldt Foundation (grant CHN/1127072).

\section{REFERENCES}

1. G. Mie, "Beiträge zur Optik trüber Medien, speziell kolloidaler Metallösungen," Ann. Phys. 25, 376-445 (1908).

2. R. J. Adrian, D. F. G. Durao, F. Durst, M. V. Heitor, M. Maeda, and J. H. Whitelaw, Laser Techniques Applied to Fluid Mechanics (Springer, 2000).

3. R. Xu, Particle Characterization: Light Scattering Methods (Kluwer Academic, 2000).

4. K. C. Neumann and S. M. Block, "Optical trapping," Rev. Sci. Instrum. 75, 2787-2809 (2004).

5. K. N. Liou, An Introduction to Atmospheric Radiation, 2nd ed. (Academic, 2002).

6. J. Guck, R. Ananthakrishnan, T. J. Moon, C. C. Cunningham, and J. Käs, "Optical deformability of soft biological dielectrics," Phys. Rev. Lett. 84, 5451-5454 (2000).

7. N. Roth, K. Anders, and A. Frohn, "Simultaneous measurement of temperature and size of droplets in micrometer range," J. Laser Appl. 2, 37-42 (1992).

8. L. P. Hsiang and G. M. Faeth, "Near-limit drop deformation and secondary breakup," Int. J. Multiphase Flow 18, 635652 (1992).

9. T. D. Taylor and A. Acrivos, "On the deformation and drag of falling viscous drop at low Reynolds number," J. Fluid Mech. 18, 466-476 (1964).

10. G. Brenn and A. Frohn, "An experimental method for the investigation of droplet oscillations in a gaseous medium," Experientia 15, 85-90 (1993).

11. W. Möbius, "Zur Theorie des Regenbogens und ihrer experimentellen Prüfung," Ann. Phys. 33, 1493-1558 (1910). 
12. C. L. Adler, J. A. Lock, and B. R. Stone, "Rainbow scattering by a cylinder with a nearly elliptical cross section," Appl. Opt. 37, 1540-1550 (1998).

13. F. Xu, K. F. Ren, X. Cai, and J. Shen, "Extension of geometrical-optics approximation to on-axis Gaussian beam scattering. II. By a spheroidal particle with end-on incidence," Appl. Opt. 45, 5000-5009 (2006).

14. Y. P. Han, L. Méès, K. F. Ren, G. Gouesbet, Z. S. Wu, G. Grehan, "Scattering light by spheroids: the far field case," Opt. Commun. 210, 1-9 (2002).

15. P. L. Marston and E. H. Trinh, "Hyperbolic umbilic diffraction catastrophe and rainbow scattering from spheroidal drops," Nature 312, 529-531 (1984).

16. P. L. Marston, "Cusp diffraction catastrophes from spheroids: generalized rainbows and inverse scattering," Opt. Lett. 10, 588-590 (1985).

17. G. Kaduchak, P. L. Marston, and H. J. Simpson, "E6 diffraction catastrophe of the primary rainbow of oblate water drops: observations with white-light and laser illumination," Appl. Opt. 33, 4691-4696 (1994).

18. J. A. Lock and F. Xu, "Optical caustics observed in light scattering by an oblate spheroid," Appl. Opt. (to be published).

19. S. Asano and G. Yamamoto, "Light scattering by a spheroidal particle," Appl. Opt. 14, 29-49 (1975).

20. J. P. Barton, "Internal and near-surface electromagnetic fields for a spheroidal particle with arbitrary illumination," Appl. Opt. 34, 5542-5551 (1995).

21. Y. Han and Z. Wu, "Scattering of a spheroidal particle illuminated by a Gaussian beam," Appl. Opt. 40, 2501-2509 (2001).

22. F. Xu, K. Ren, G. Gouesbet, G. Gréhan, and X. Cai, "Generalized Lorenz-Mie theory for an arbitrarily oriented, located, and shaped beam scattered by a homogeneous spheroid," J. Opt. Soc. Am. A 24, 119-131 (2007).

23. B. van der Pol and H. Bremmer, "Diffraction of electromagnetic waves from an electrical point source round a finitely conducting sphere with applications to radiotelegraphy and the theory of the rainbow I," Philos. Mag. 24, 141-176, (1937)

24. B. van der Pol and H. Bremmer, "Diffraction of electromagnetic waves from an electrical point source round a finitely conducting sphere with applications to radiotelegraphy and the theory of the rainbow II," Philos. Mag. 24, 825-864, (1937).

25. G. Gouesbet, "Debye series formulation for generalized Lorenz-Mie theory with the Bromwich method," Part. Part. Syst. Charact. 20, 382-386 (2003).
26. P. Debye, "Das elektromagnetische Feld un einen Zylinder und die Theorie des Regenbogens," Phys. Z. 9, 775-778 (1908), translated and reprinted in Selected Papers on Geometrical Aspects of Scattering, P. L. Marston, ed., SPIE Milestone Series Vol. MS89 (SPIE, 1994), pp 198-204.

27. J. A. Lock and C. L. Adler, "Debye-series analysis of the first-order rainbow produced in scattering of a diagonally incident plane wave by a circular cylinder,” J. Opt. Soc. Am. A 14, 1316-1328 (1997).

28. J. A. Lock, "Scattering of an electromagnetic plane wave by a Luneburg lens. II. Wave theory," J. Opt. Soc. Am. A 25, 2980-2990 (2008)

29. R. Li, X. Han, H. Jiang, and K. F. Ren, "Debye series of normally incident plane-wave scattering by an infinite multilayered cylinder," Appl. Opt. 45, 6255-6262 (2006).

30. F. Xu, K. Ren, and X. Cai, "Expansion of arbitrarily oriented, located and shaped beam in spheroidal coordi-nates," J. Opt. Soc. Am. A 24, 109-118 (2007).

31. J. A. Lock and E. A. Hovenac, "Assessing the contributions of surface waves and complex rays to far-field Mie scattering by use of the Debye series," J. Opt. Soc. Am. A 9, 781795 (1992).

32. J. A. Lock, "Cooperative effects among partial waves in Mie scattering," J. Opt. Soc. Am. A 5, 2032-2044 (1988).

33. G. M. Hale and M. R. Querry, "Optical constants of water in the 200-nm to $200-\mu \mathrm{m}$ wavelength region," Appl. Opt. 12, 555-563 (1973).

34. J. A. Lock, "Supernumerary spacing of rainbows produced by an elliptical-cross-section cylinder. I. Theory," Appl. Opt. 39, 5040-5051 (2000).

35. H. C. van de Hulst, Light Scattering by Small Particles (Dover, 1981), Chap. 13

36. R. T. Wang and H. C. van de Hulst, "Rainbows: Mie computations and the Airy approximation," Appl. Opt. 30, 106117 (1991).

37. J. Nocedal and S. J. Wright, Numerical Optimization (Springer-Verlag, 1999), Chap. 13.

38. J. A. Lock, "Scattering of an electromagnetic plane wave by a Luneburg lens. I. Ray theory," J. Opt. Soc. Am. A 25, 2971-2979 (2008)

39. D. S. Langley and M. J. Morrell, "Rainbow-enhanced forward and backward glory scattering," Appl. Opt. 30, 34593467 (1991).

40. S. Holler, Y. L. Pan, R. K. Chang, J. R. Bottiger, S. C. Hill, and D. B. Hillis, "Two-dimensional angular optical scattering for the characterization of airborne microparticles," Opt. Lett. 23, 1489-1491 (1998). 